\title{
Glottopol
}

Revue de sociolinguistique en ligne

$33 \mid 2020$

Varia - Liberté, égalité, diversité !

\section{La prononciation du français parlé en Côte-d'Ivoire}

\section{Assémou Maurice Ludovic Assémou}

\section{OpenEdition}

Journals

Édition électronique

URL : https://journals.openedition.org/glottopol/616

DOI : 10.4000/glottopol.616

ISSN : 1769-7425

\section{Éditeur}

Presses universitaires de Rouen et du Havre

\section{Référence électronique}

Assémou Maurice Ludovic Assémou, «La prononciation du français parlé en Côte-d'Ivoire », Glottopol [En ligne], 33 | 2020, mis en ligne le 01 janvier 2020, consulté le 02 octobre 2021. URL : http:// journals.openedition.org/glottopol/616 ; DOI : https://doi.org/10.4000/glottopol.616 


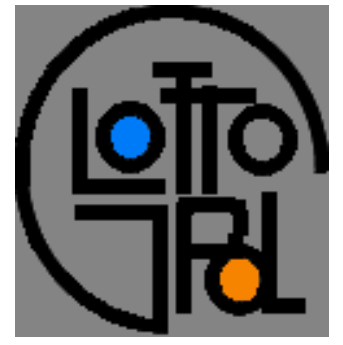

\section{GLOTTOPOL}

Revue de sociolinguistique en ligne $n^{\circ} 33$ - février 2020

Varia : Liberté, égalité, diversité !

\section{SOMMAIRE}

Hommages à Jean-Baptiste Marcellesi : À ringrazià vi, par Niculau Sorba ; “Ciao Ziu ! » par Philippe Blanchet.

Clara Mortamet : Présentation du numéro : Liberté, égalité, diversité !

Manon Him-Aquilli : "Prendre la parole sans prendre le pouvoir ». La mise en registre de l'horizontalité dans les assemblées générales anarchistes/autonomes.

Siham Hocini : La formulation du désaccord dans les débats télévisés algériens : quels "effetsde-politesse "?

Pauline Rannou : Trajectoires de la surdité en France. Parents entendants d'enfants sourds : questionner la fragmentation de l'identité sourde.

Catherine Combaz-Champlaine: Les rectifications orthographiques de 1990 comme révélateurs du rapport des enseignants à l'orthographe.

Marie-Madeleine Bertucci : Éléments d'épistémologie pour l'étude des espaces ségrégués des banlieues urbaines sensibles de la région parisienne.

Élise Gandon: Usages du numérique et illectronisme: nouvelles tâches pour le lecteurscripteur, nouveaux enjeux d'apprentissage.

Marc Arabyan : Quelle langue pour la recherche neurolinguistique française?

José R. de Arellano : Le rapprochement entre l'espagnol et le portugais pendant la décennie dorée sud-américaine : une problématique de l'horizontalité supercentrale.

Adam Wilson: Normes interactionnelles globalisées et communautés de pratique discontinues : les dynamiques sociolinguistiques du tourisme international.

Mélanie Lancien : Caractérisation de la variation liée à la situation de communication : apport de l'acoustique à la phonostylistique.

Assémou Maurice Ludovic Assémou : La prononciation du français parlé en Côte d'Ivoire. 


\title{
LA PRONONCIATION DU FRANÇAIS PARLÉ EN CÔTE D'IVOIRE
}

\author{
Assémou Maurice Ludovic ASSÉMOU \\ Université Félix Houphouët-Boigny (Abidjan, Côte d'Ivoire)
}

\section{Introduction}

La Côte d'Ivoire est souvent citée en exemple quand l'on aborde les questions de dynamique et d'appropriation de la langue française. Manessy (1993), par exemple, fait remarquer que c'est l'un des rares pays africains où le nombre des francophones l'emporte sur celui des lettrés, et où l'on entend parler français dans les rues. L'ancien Secrétaire général de l'Organisation internationale de la Francophonie, Abdou Diouf, lui, met en exergue les contributions ivoiriennes à la vitalité du français, quand il dit :

[...] la langue française doit féliciter tous les Ivoiriens pour leur imagination, leur manière savoureuse de s'exprimer en français. Chaque fois qu'on demande de donner des expressions qui ne sont pas venues de l'Hexagone ou des pays du Nord, les exemples qui viennent à l'esprit, c'est des exemples ivoiriens. ${ }^{l}$

Cependant, les particularités qui font du français parlé en Côte d'Ivoire une langue «savoureuse » ne sont pas du gout de certains Ivoiriens (notamment quelques puristes, professionnels de la langue française) qui les considèrent comme des altérations, de mauvais usages, qui menacent la vie de la belle langue. On devine alors le but de ces émissions du type « Ne dites pas...dites plutôt... », diffusées sur les chaines de radio et de télévision nationales. Vraisemblablement, ces émissions n'atteignent pas leur but, puisque le français continue de se particulariser en Côte d'Ivoire, et cela est compréhensible : le changement est aussi le lot des langues vivantes, et le français ne saurait y déroger.

D'un autre côté, les particularités du français parlé en Côte d'Ivoire en ont fait un véritable phénomène scientifique. Ses aspects sociolinguistiques et linguistiques ont donné lieu à une riche littérature (Hattiger, 1983 ; Simard, 1994, 1998 ; Lafage, 1998, 2002 ; Boutin, 2002 ; Aboa, 2008 ; Kouadio, 2006, 2008 ; Assémou, 2017, 2018 ; etc.). En la matière, le domaine phonético-phonologique, très peu abordé, reste le parent pauvre de la description. Les travaux existants concernent beaucoup plus l'inventaire des sons. L'étude de Boutin et Turcsan (2009) sur la prononciation du français de Côte d'Ivoire montre que l'inventaire consonantique comprend des affriquées, des clics, des prénasalisées et des consonnes à double articulation, qui s'ajoutent aux consonnes françaises. Ils relèvent une ressemblance entre le système

\footnotetext{
${ }^{1}$ Discours à la $39^{\mathrm{e}}$ session de l'Assemblée parlementaire de la Francophonie. Extrait tiré du Journal télévisé de 13h sur RTI1 (première chaine de télévision publique nationale en Côte d'Ivoire) le 10 juillet 2013.
} 
vocalique du français de Côte d'Ivoire et celui du français standard, à la différence que, dans le système ivoirien, la voyelle postérieure orale [a] est absente, et que quelques voyelles nasales inconnues en français standard sont à prendre en compte dans l'inventaire. Sur le plan du fonctionnement des sons, toujours selon ces auteurs, le français de Côte d'Ivoire est marqué par la disparition de $\mathrm{R}$, la vélarisation de $\mathrm{L}$, des réductions de groupes consonantiques qui "s'inscrivent dans une logique de simplification et de réduction des contrastes en position faible ». Dans son étude sur la prosodie du français de Côte d'Ivoire, Dodo (s.d) montre que chez les francophones ivoiriens, le [u] est moins postérieur et tend à se centraliser, le [i] plus antérieur et moins fermé que [y], et le [o] plus ouvert, un peu plus postérieur.

D'une manière générale, les travaux n'insistent pas sur l'aspect fonctionnel des sons, qui, pourtant, laisse apparaitre des faits dignes d'intérêt. Ces faits partent de la simple modification du timbre de certaines voyelles à des altérations complètes de certains mots ou phrases, rendant ces unités difficilement reconnaissables. Ces modifications concernent, à des degrés divers, la quasi-totalité des locuteurs francophones de ce pays.

Si la prononciation approximative d'un francophone peu ou pas lettré peut, à la limite, sembler normale, le fait que des individus diplômés de l'enseignement secondaire ou supérieur soient concernés par les mêmes phénomènes soulève quelques interrogations dont celle-ci : comment les particularités phonétiques du français parlé éclairent-elles la dynamique de la langue française en Côte d'Ivoire ?

Cet article s'intéresse particulièrement au fonctionnement des sons du français parlé en Côte d'Ivoire. Alors que la plupart des travaux mettent en avant le rôle prépondérant des langues maternelles ivoiriennes dans la particularisation du français parlé, nous montrons ici que ces langues ne sont pas responsables de tous les changements phonétiques qui surviennent (et s'installent) dans le français oral. L'étude fait ainsi ressortir qu'on peut rendre compte des modifications observées à partir de lois phonétiques universelles.

Nous commencerons par un exposé de la démarche méthodologique, qui sera le lieu de présenter notre terrain d'enquête, l'échantillon d'étude, ainsi que la procédure d'analyse des données. Cette première section sera suivie d'une autre consacrée aux résultats. Nous y ferons un inventaire des sons avant d'analyser leur fonctionnement. Dans la dernière section qui discute les résultats, nous tenterons d'expliquer les différentes particularités observées.

\section{Méthodologie}

La réalisation de cette étude a nécessité une phase d'enquête au cours de laquelle nous nous sommes intéressé aux productions orales de personnes issues de la population ivoirienne. Ce sont ces productions qui nous ont servi de base d'analyse. Le terrain d'étude présente cependant un aspect quelque peu particulier.

\section{Terrain d'enquête}

Le sens commun considère le terrain (presque) exclusivement comme un lieu physique, un espace géographique. Cette définition est quelque peu restrictive, car :

Le terrain n'est pas uniquement ni principalement une aire territoriale, un espace géographique, un lieu où se produisent des phénomènes humains et sociaux. [...] Un terrain, c'est avant tout un ensemble d'interactions [...] entre un chercheur et d'autres personnes. [...] Et l'on peut envisager des interactions indirectes telles que celles qui se produisent ordinairement à travers la communication écrite, lorsqu'on travaille sur des témoignages historiques ou des textes littéraires ou encore sur des documents médiatiques comme la publicité, la signalisation, la presse, la radio, les graffitis, la télévision, le cinéma, des sites ou des forums internet, etc. (Blanchet, 2012 : 31) 
Comme on peut le voir avec Blanchet, la notion de «terrain» recouvre une variété de champs d'investigation que la définition générale du terme ne permet pas d'appréhender. Le rappel de cette définition présente donc une certaine utilité pour notre propos, dans la mesure où le terrain de cette étude ne correspond pas à un lieu physique ou un espace géographique. L'étude s'appuie en effet sur des documents médiatiques issus de la radio, de la télévision et d'Internet. Le corpus est constitué de productions orales extraites d'émissions radiophoniques, d'émissions humoristiques, de chansons d'artistes ivoiriens, d'interviews et discours d'hommes et de femmes politiques ivoiriens, ainsi que de films et interviews de comédiennes ivoiriennes. En dehors des émissions radiophoniques que nous avons enregistrées nous-même, toutes les autres sources citées ici sont disponibles sur Internet. Les différents intervenants dans ces clips (artistes, hommes et femmes politiques, comédiennes) sont bien connus en Côte d'Ivoire.

Le choix de ces documents médiatiques se justifie par le fait qu'ils sont révélateurs, d'une part, de cette appropriation spectaculaire du français en Côte d'Ivoire dont parlent certains chercheurs (Canut, 2006 ; Manessy, 1993 ; Kouadio, 2008 ; Simard, 1994, etc.) et, d'autre part, de l'usage décomplexé que font les Ivoiriens de la variété non-standard du français qui leur sert de principale langue d'intercompréhension. On retrouve aisément dans ces documents le français tel qu'il est couramment pratiqué en Côte d'Ivoire. En écoutant ces documents, on se rend vite compte en effet que le français parlé n'est pas (toujours) la norme standard de référence, mais plutôt une forme qui s'en éloigne plus ou moins. Cette réalité permet de soutenir Kouadio (2008) qui dit que le français est passé d'une langue coloniale-impériale à une langue ivoirienne dont la pratique décomplexée s'observe à travers l'usage de variétés ${ }^{2}$ plus ou moins proches du français central. Ceci ne semble d'ailleurs pas étonner l'auteur qui pense que, sans doute sous l'influence d'Houphouët-Boigny (premier président ivoirien) «qui a donné au français un rôle essentiellement pratique [...], parler "le français de France" n'a probablement jamais connu en Côte d'Ivoire le même prestige que dans d'autres pays africains francophones comme par exemple le Sénégal, le Gabon ou le Congo Brazzaville ». Nombre de chercheurs parlent de l'existence d'un « français de Côte d'Ivoire » ou « français ivoirien » qui se définit par rapport à un continuum allant d'une variété basilectale à un français acrolectal, en passant par un mésolecte et un argot.

Dans la pratique, ces différentes variétés s'interpénètrent. Cependant, alors qu'on assiste à une quasi-disparition de l'acrolecte ${ }^{3}$, un français moyen résultant du mélange de toutes les variétés en présence se répand largement et est utilisé comme langue véhiculaire ${ }^{4}$. En considérant la sphère musicale, ce français ivoirisé est la langue du zouglou et du rap abidjanais, deux genres musicaux particulièrement engagés dans la dénonciation des maux de la société ivoirienne. Mais, il n'y a pas que la musique. Dans les productions humoristiques, à la télévision (émissions, séries ivoiriennes, etc.), à la radio, dans les meetings politiques, etc., on retrouve ce même français, qui apparait comme l'outil linguistique idéal quand il s'agit de s'adresser aux masses populaires. Aussi peut-on considérer ces sources comme des terrains dignes d'intérêt quand il s'agit d'étudier le français parlé en Côte d'Ivoire.

\section{Échantillon}

L'échantillon d'étude est composé de vingt et un (21) individus, dont sept (07) femmes et quatorze (14) hommes, issus des 4 grandes familles linguistiques (kwa, kru, gur et mandé (nord et sud)) qui regroupent les langues maternelles ivoiriennes. Ces individus, que nous désignons

\footnotetext{
${ }^{2}$ Sur la question des variétés du français parlé en Côte d'Ivoire, on pourra se référer à Hattiger (1983), Mel et Kouadio (1990), Simard (1994, 1998), Lafage (1998, 2002), Ploog (2001), Boutin (2002), Aboa (2008), Kouadio (2006, 2008), Assémou (2017, 2018), entre autres.

${ }^{3}$ Sur la question de la quasi-disparition de l'acrolecte, on pourra se reporter à Boutin (2002).

${ }^{4}$ Selon Dumont (1990 : 23-24) « une langue devient vraiment véhiculaire lorsqu'elle est utilisée par des locuteurs libérés de toute contrainte normative acceptant d'en pratiquer une forme abâtardie, aux yeux des puristes ».
} 
par "participant(e)s », sont de catégories socioprofessionnelles et de niveaux d'instruction différents. Les niveaux d'instruction sont: « Non scolarisé », "Primaire », " Secondaire » et «Bac + ». Les participants identifiés comme «Non scolarisés » sont ceux qui n'ont été ni scolarisés ni alphabétisés. Dans l'échantillon, deux vendeuses répondent à ce critère. Nous avons recueilli leurs interventions à la radio. Les individus de niveau «Primaire » ne sont pas allés au-delà de la sixième année du cycle primaire. Ceux de niveau «Secondaire » ont le baccalauréat tout au plus. Les participants de niveau «Bac + », diplômés de l'enseignement supérieur, sont les plus nombreux (plus de $60 \%$ de l'échantillon). Ce sont des anciens étudiants, des enseignants-chercheurs, etc.

La situation socioprofessionnelle fait référence à la dernière activité professionnelle (publiquement connue) de l'individu. Sur la base de ces variables, on peut répartir l'échantillon comme suit :

- Selon 1'appartenance linguistique :

- Kwa : 07

- Kru : 04

- Gur : 03

- Mandé : 07 (Nord : 02 ; Sud : 05)

- Selon la situation socioprofessionnelle et les niveaux d'instruction :

- 05 enseignants-chercheurs de niveau « Bac + » (1 ancien président, 2 ministres, 1 député, 1 maire, et 1 défenseur des droits de l'homme)

- 1 député de niveau « $\mathrm{Bac}+»$

- 2 ministres de niveau « $\mathrm{Bac}+»$

- 1 journaliste de niveau « $\mathrm{Bac}+»$

- 4 artistes musiciens dont 02 « $\mathrm{Bac}+», 01$ «Secondaire » et 01 «Primaire »

- 3 humoristes de niveau « $\mathrm{Bac}+»$

- 3 comédiennes (niveaux inconnus)

- 2 vendeuses dans l'informel « Non scolarisées »

\section{Analyse des données}

Les émissions radiophoniques et les clips vidéo dont nous nous sommes servi dans la constitution du corpus ont une durée totale de cent quatre-vingt-dix-huit minutes douze secondes (198 minutes 12 secondes), soit trois (3) heures dix-huit (18) minutes et douze (12) secondes. L'analyse des données repose sur une approche contrastive. En écoutant les différents documents qui composent notre corpus, nous avons relevé les unités lexicales dont la prononciation ne correspondait pas à celle admise en français standard. Ces unités n'ont toutefois pas été totalement isolées de leurs contextes linguistiques. Cette précaution nous a semblé nécessaire du fait que, dans certains cas, l'environnement peut induire certaines modifications, notamment en raison du phénomène de la coarticulation.

Pour chaque donnée à analyser, nous donnons, à gauche, une transcription phonétique (entre crochets droits), au centre, une transcription orthographique (en italique), et, à droite, une glose (entre guillemets). Les mots qui apparaissent en italique dans les gloses sont des mots nouchi (l'argot ivoirien), ou des emprunts aux langues maternelles ivoiriennes. Pendant la phase de transcription phonétique, l'Interactive IPA Chart, une version sonore de l'Alphabet Phonétique international, nous a été d'une grande utilité. Nous y avons recouru pour identifier certains sons, notamment chez une participante non scolarisée. 


\section{Résultats}

Diverses particularités apparaissent aussi bien dans l'inventaire des sons que dans leur fonctionnement.

\section{Inventaire des sons}

L'inventaire des sons fait apparaitre des unités inconnues de la langue standard, tant en ce qui concerne les voyelles que pour ce qui est des consonnes.

\section{Les consonnes du français parlé en Côte d'Ivoire}

Les données recueillies montrent que le système consonantique est un peu plus étendu que celui du français standard. En plus des consonnes françaises, nous avons relevé les occlusives labio-vélaires [gb] (1a) et [kp] $(1 \mathrm{~b}, \mathrm{c})$, les affriquées [d马] $(2 \mathrm{a}, \mathrm{b})$ et [tg] (2c), ainsi que l'occlusive palatale [J] (3) :
(1) a. [pehegboflotopului]
péher gbofloto pour lui
« payer gbofloto pour lui »
b. [døbonpwanedakpi]
deux bonnes poignées d'akpi
« deux bonnes poignées d'akpi »
c. [isõakrofeõdirعakpanĩ]
$i$ sont accrochés on dirait akpani
(2) a. [sєnuõsãaḑovã] c'est nous on sent adjovan
b. [wolososepadłãdzu] wolosso c'est pas djandjou
c. [nuveltfu:z]
nouvelle tchouse
« ils sont accrochés, on dirait des akpani »
« c'est nous qui puons l'adjovan »
" une Wolosso n'est pas une djandjou »
« nouvelle tchouse »
(3) [sekejevãajovã]
cé qué yé vends ? aguiovan...
«ce que je vends? aguiovan... »

Ce sont des mots tels que " gbofloto », «akpi », «akpani », «adjovan », « djandou », « tchouse », et « aguiovan » qui justifient l'extension du système consonantique. Étant donné qu'ils sont inconnus en français standard, nous en donnons les traductions ci-dessous :

\begin{tabular}{|c|c|c|c|}
\hline \multirow{2}{*}{ Consonnes } & \multicolumn{2}{|l|}{ Exemples de mots } & \multirow{2}{*}{ Glose } \\
\hline & Forme orthographique & Prononciation & \\
\hline [gb] & Gbofloto & [gbofloto] & Galette de farine blé \\
\hline \multirow{2}{*}{ [kp] } & Akpi & [akpi] & Amande du Ricinodendron heudelotii \\
\hline & Akpani & [akpanĩ] & Chauve-souris \\
\hline \multirow[t]{2}{*}{ [d3] } & Adjovan & [aḑ̧ovã] & $\begin{array}{l}\text { Poisson salé, qu'on laisse faisander et sécher, } \\
\text { utilisé comme assaisonnement }\end{array}$ \\
\hline & Djandjou & [d]ãḑu] & Prostituée \\
\hline$[\mathrm{t} f]$ & Tchouse & [tfuz] & Chaussure \\
\hline [J] & Aguiovan & [ałovã] & (variante de « adjovan $»)$ \\
\hline
\end{tabular}

Tableau 1 : Illustration des consonnes non attestées en français standard

En ce qui concerne la prononciation $d u$ « $R$ », les variantes apicale $[r]$ et uvulaire $[\mathrm{R}] \mathrm{du}$ français standard sont toutes deux attestées. Nous avons cependant noté une prépondérance du « R » apical. Sur les vingt et un (21) participants, seize (16), soit 76,19\% de l'échantillon, réalisent ce $《 R$ », contre seulement 9,52\% pour la variante uvulaire. Nous avons également observé des cas d'alternance des deux variantes dans le discours, notamment chez deux (2) des participants. En outre, chez une participante «Non scolarisée » dont la langue maternelle appartient à la famille kwa, nous avons relevé un autre « $\mathrm{R}$ » plutôt proche de [1]. Une recherche de correspondance dans l'Interactive IPA Chart nous a permis de nous rendre compte qu'il 
s'agit de la consonne alvéolaire battue [r]. Ci-dessous la transcription d'une phrase dans laquelle apparait cette consonne :

(4) [3egããpealepehediri / prepare / donemezãfã] Jé gan en pé aller péher di riz, préparer, donner mes enfants

L'énoncé (4) correspond à peu près à «Je gagne un peu (d'argent) pour aller payer du riz que je prépare pour (le donner à) mes enfants ». Si l'on compare cet énoncé à sa glose, on peut se rendre compte que le français de cette participante est très approximatif; ce qui reflète sa non-scolarisation.

Sur la base de ces constatations, on peut établir les deux tableaux de consonnes suivants :

\begin{tabular}{|l|c|c|l|c|c|c|}
\hline & \multicolumn{6}{l|}{ OCCLUSIVES } \\
\hline Mode Lieu & Bilabiales & Alvéolaire & Dorso-palatale & Dorso-vélaires & Labio-vélaires & Palatale \\
\hline Sourdes & $\mathrm{p}$ & $\mathrm{t}$ & & $\mathrm{k}$ & $\mathrm{kp}$ & \\
\hline Sonores & $\mathrm{b}$ & $\mathrm{d}$ & & $\mathrm{g}$ & $\mathrm{gb}$ & $\mathrm{J}$ \\
\hline Nasales & $\mathrm{m}$ & $\mathrm{n}$ & $\mathrm{n}$ & $\mathrm{g}$ & & \\
\hline
\end{tabular}

Tableau 2 : Tableau des occlusives

Tout comme en français standard, les mots dans lesquels apparait la consonne [ $\mathrm{y}$ ] sont des emprunts à l'anglais.

\begin{tabular}{|l|c|c|c|c|l|l|}
\hline & \multicolumn{2}{l|}{ CONSTRICTIVES } & AFFRIQUÉES \\
\hline Mode Lieu & $\begin{array}{l}\text { Labio- } \\
\text { dentales }\end{array}$ & Alvéolaires & $\begin{array}{l}\text { Post- } \\
\text { alvéolaire }\end{array}$ & $\begin{array}{l}\text { Apico- } \\
\text { alvéolaires }\end{array}$ & Uvulaire & Palatales \\
\hline Sourdes & $\mathrm{f}$ & $\mathrm{s}$ & $\mathrm{f}$ & & & $\mathrm{t}$ \\
\hline Sonores & $\mathrm{v}$ & $\mathrm{z}$ & 3 & & & $\mathrm{~d}$ \\
\hline Latérale & & & & 1 & & \\
\hline Roulées & & & & $\mathrm{r}$ & $\mathrm{R}$ & \\
\hline Battue & & & & $\mathrm{r}$ & & \\
\hline
\end{tabular}

Tableau 3 : Tableau des constrictives et des affriquées

Nous avons relevé vingt-cinq (25) sons consonnes, dont six (6) non attestés en français standard. D'un point de vue phonologique, on peut affirmer que c'est un système à vingt-deux (22) phonèmes consonantiques, étant donné, d'une part, que les variantes de « $\mathrm{R}$ » (apicale, uvulaire, battue) identifiées n'ont pas de rôle phonémique et, d'autre part, que pour [dz] et [f] tout semble indiquer qu'il s'agit de deux variantes d'un même phonème.

\section{Les voyelles du français parlé en Côte d'Ivoire}

Les données suggèrent que la voyelle postérieure orale /a/ et sa correspondante nasale /ãa ne sont pas attestées dans le système vocalique ivoirien. Aucun des participants en effet ne réalise ces deux voyelles. Cependant, nous ne pouvons pas affirmer qu'il y a une neutralisation de l'opposition /a/ / /a/ comme en français standard. Aucune preuve ne permet d'attester l'existence antérieure de la postérieure orale /a/ dans le français des locuteurs. Quant à la postérieure nasale [ã], les participants lui substituent l'antérieure nasale [ã]. Ainsi, «avant» /avã/ et «maman »/mamã/, par exemple, sont réalisés respectivement [avã] et [mãmã] comme dans l'énoncé (5) suivant :

(5) [avãnomãmãpotepan] avant nos mammans portaient pagnes « avant nos mamans portaient des pagnes » 


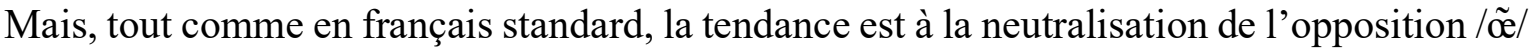

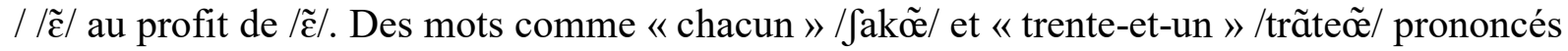

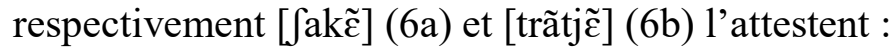

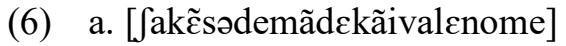

b. [lətrãtjẽ sktıbdømildiset] chaquin $^{5}$ se démandait quand $i$ va les nommer

le trentien octob' deux mille dix-sept
« chacun se demandait quand est-ce qu'il allait les nommer »

« le trente-et-un octobre deux mille dix-sept »

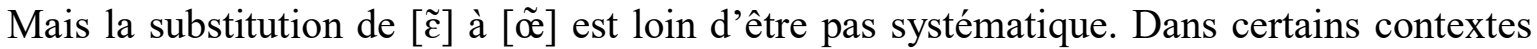
comme en (7) ci-dessous, la voyelle [ã] est bel et bien réalisée :
(7) a. [ləvõnœfdesãbr]
le vungt-neuf décembre
« le vingt-neuf décembre »
b. [Jakõdspa:rtimãb]
chacun des partis memb'
« chacun des partis membres »

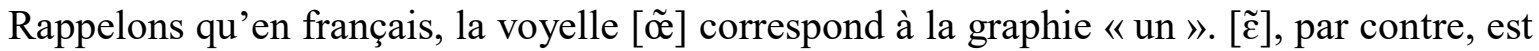
la forme phonétique de diverses formes graphiques : « in » (fin), « ain » (main), « aim » (daim),

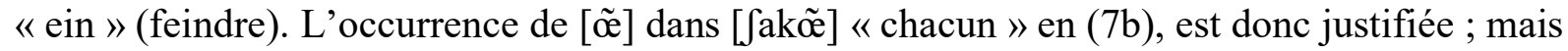

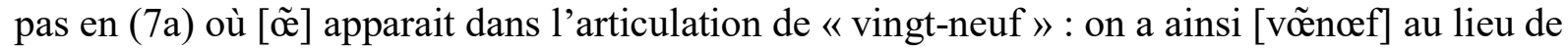

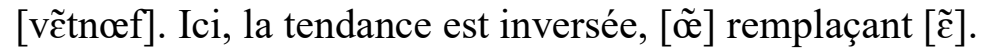

Au-delà de ces observations, des mots tels que «gbonhin » [gbõhĩ] (8a) et «tchoun » [t fũ] (8b) observables dans le français de certains des participants pourraient justifier l'inclusion des voyelles nasales [ĩ] et [ũ] dans l'inventaire. Considérons les énoncés de (8) :

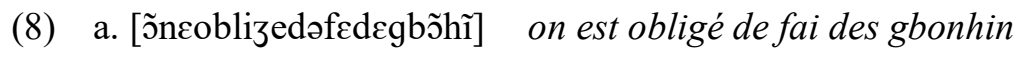

b. [ozoduitytatfũ]

aujourd'hui tu te tchoun
« nous sommes obligés de constituer des groupes »

« aujourd'hui, tu te vends »

Les données montrent en définitive que toutes les voyelles françaises, à l'exception de [a] et [ã], apparaissent dans le français des participants. Par ailleurs, ces mêmes données suggèrent que trois voyelles nasales non-attestées en français standard, à savoir [ã ; ĩ ; ũ], sont à prendre en compte dans l'inventaire. L'ensemble des voyelles peut être présenté comme ci-dessous :

\begin{tabular}{|c|c|c|c|c|}
\hline \multirow{3}{*}{ Aperture Mode d'articulation } & \multicolumn{4}{|c|}{ Lieu d'articulation } \\
\hline & \multicolumn{2}{|c|}{ Antérieures } & \multirow{2}{*}{$\begin{array}{l}\text { Centrales } \\
\text { Neutres }\end{array}$} & \multirow{2}{*}{$\begin{array}{l}\text { Postérieures } \\
\text { Arrondies }\end{array}$} \\
\hline & Etirées & Arrondies & & \\
\hline Fermées & $\mathrm{i} / \tilde{\mathrm{i}}$ & $\mathrm{y}$ & & $\mathrm{u} / \tilde{\mathrm{u}}$ \\
\hline Mi-fermées & e & $\varnothing$ & & o \\
\hline Mi-ouvertes & $\varepsilon / \tilde{\varepsilon}$ & $œ / \tilde{œ}$ & ə & o/ $\tilde{\jmath}$ \\
\hline Ouvertes & $\mathrm{a} / \tilde{a}$ & & & \\
\hline
\end{tabular}

Tableau 4 : Tableau des voyelles

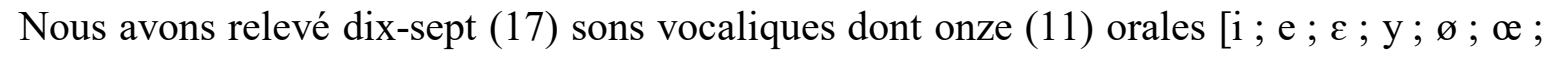

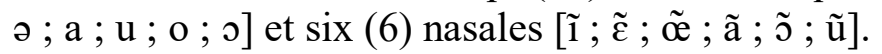

\section{Fonctionnement des consonnes}

Le fonctionnement consonantique est marqué par divers phénomènes dont des chutes.

\footnotetext{
${ }^{5}$ Nous employons « qu » en lieu et place de « $\mathrm{c} »$ ici pour éviter que le mot soit lu avec un « s » phonétique.
} 


\section{Chutes des consonnes [1] et [r] en position finale}

Devant un verbe à initiale consonantique, les participants ont tendance à ne pas réaliser le [1] final des pronoms «il(s)»/i1/ et « elle(s)»/عl/. Leurs formes phoniques respectives [il] et $[\varepsilon l]$ ne sont plus réduites qu'aux voyelles qu'elles contiennent :

(9) a. [petinjarafolosenufo / pétit nyarafolo Sénoufo, quand « le petit nyarafolo Sénoufo, quand il dit
kãidinõsєnว̃]
$i$ dit non c'est non
non, c'est non »
b. [is̃̃wit / izõdømikro]
i sont huit, i z'ont deux micros « ils sont huit, ils ont deux micros »
c. [kã $r$ rãt / sckomsa]
quand è rent', c'est comme ça « quand elle rentre, c'est comme ça »
d. [omyluøevamet
au mulueu è va mett' son
sõfamøadzovã]
fameux adjovan
« au milieu, elle va mettre son fameux adjovan»

Mais, en cas d'enchainement, le [1] est maintenu dans la prononciation de ces pronoms :

[ilelatisepa] il est là ti sais pas

« il est là mais tu ne le sais pas »

Notons que c'est dans l'articulation de « il(s) » et « elle(s)» que nous observons la chute de [1] en fin de mot. L'énoncé (11), par exemple, montre que les participants réalisent effectivement le [1] final dans l'articulation des autres mots français :

$$
\text { [õlapellidvokal] on l'appelle lead vocal « on l'appelle lead vocal» }
$$

Il faut cependant distinguer entre finale syllabique et fin de mot. Si [1] est conservé en fin de mot (hormis «il(s) et « elle(s)»), cette consonne tombe généralement en finale syllabique ; ce

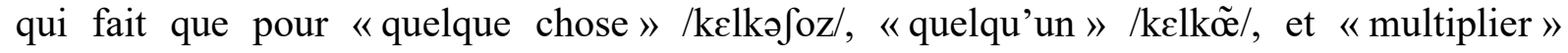

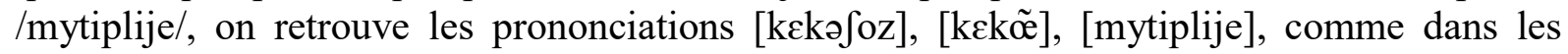
exemples de (12):

(12) a. [saresãbpaakekəðozsa]

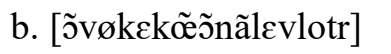

c. [tusəramytiplijepadø] ça ressemb' pas à quêque chose ça?

on veut quèqu'un on enlève l'autre

tout sera mutiplié pa deux
« ça ne ressemble pas à quelque chose, ça?»

« on veut quelqu'un, on enlève l'autre »

« tout sera multiplié par deux »

La consonne $[\mathrm{r}]$ ne distingue pas entre finale syllabique et fin de mot: dans les deux contextes, elle peut être élidée. Analysons le corpus ci-dessous :

(13) a. [ticrasazjealotypa:1]

b.

c.

[regadmamitmãmãprãpup prend pour préparer repare]

la Cô'd'Ivoi serait

dévéloppée [lakodivwasəredevelope]

ti es rassasié alo tu paales

régade mamite maman

d. [õmaãvwajealynivesite] on m'a envoyé à l'univessité « on m'a envoyé à l'Université »
«Tu es rassasié, alors tu parles »

« la Côte d'Ivoire serait développée » « regarde la marmite que maman utilise pour cuisiner »

En (13a) et (13b), [r] est en finale de mot dans "alors»/als:R/ et "Côte d'Ivoire » /kotdivwa:R/. En (13c) et (13d), la position de [r] dans «marmite »/ma:rmit/ et « université » /ynive:rsite/ correspond à une finale syllabique qui ne coïncide pas avec la fin des mots. Mais, on remarque qu'aussi bien en $(13 a, b)$ qu'en $(13 c, d)$, la consonne [r] tombe malgré la différence 
des contextes. La chute de la liquide [r] semble donc s'étendre à toutes ses positions, excepté la position initiale syllabique.

\section{Réduction des groupes consonantiques}

En français standard, en positions initiale, médiane et finale, $[\mathrm{r}]$ peut former des groupes avec les occlusives $[\mathrm{p} ; \mathrm{b} ; \mathrm{t} ; \mathrm{d} ; \mathrm{k} ; \mathrm{g}]$ et les fricatives [f; $\mathrm{f}]$. [l] pour sa part est exclu après [d] et $[\mathrm{t}]$. Dans les groupes « Consonne +1 » (désormais [Cl]) et «Consonne $+\mathrm{r}$ » (désormais $[\mathrm{Cr}]$ ) en position finale syllabique, la non-réalisation des liquides [r] et [1] semble être la règle. Les groupes $[\mathrm{Cl}]$ et $[\mathrm{Cr}]$ sont le plus souvent réduits à $[\mathrm{C}]$, c'est-à-dire la consonne qui précède $[\mathrm{r}]$ ou $[1]$ :

(14) a. [sivuzazutesãswasãkat]

b. [vuzavelazãepuinuõsuf]

c. [aferasuiv]

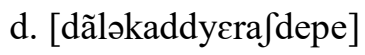

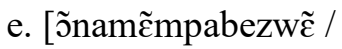
niẽteredəfєpalababedje]

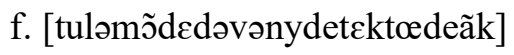

g. [scprop]

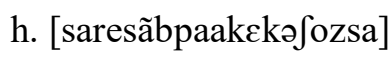

i. [ع̃nєzãpkivjẽdəlakədivwa:]

j. [sclepetimaladiepuiõreg]

k. [ekivjẽməprãd / mõnõk] si vous ajoutez cent soixanquat'

vous avez l'agent et puis nous on souff

affaire à suiv'

dans le cad' du RHDP

on n'a même pas bésoin $n i$ intérêt de fai palab'à Bédié

tout le monde est devenu détecteur dé enc'

c'est prop'!

ça ressemb' pas à quêque chose ça?

in èzemp' qui vient de la Co' d'Ivoii

c'est les péti' maladies et puis on règ'

et qui vient me prend'? Mon onc'!
« si vous ajoutez cent soixante-quatre »

« vous avez l'argent et puis nous on souffre »

« affaire à suivre »

« dans le cadre du RHDP »

« on n'a même pas besoin ni intérêt de faire palabre à

Bédié »)

« tout le monde est devenu détecteur d'encre »

«c'est parfait!»

« ça ne ressemble pas à quelque chose, ça ?»

« un exemple qui vient de la Côte d'Ivoire »

« ce sont les petites maladies et puis on règle »

« et qui vient me prendre?

Mon oncle!»

On remarque que les positions initiale et médiane préservent l'intégrité des groupes [Cr] et [Cl]. C'est en finale de mot que se produit la réduction des groupes. Dans les exemples (14a$\mathrm{g})$, les groupes [Cr] en fin de mots sont réduits à [C] seulement dans « quatre » [kat], " souffre » [suf], « suivre » [suiv], « cadre » [kad], « palabre » [palab], « encre » [ãk] et " propre » [prop]. Il en est de même dans les exemples de (14h-k) qui illustrent les cas de [1]. On observe la chute de cette consonne dans « ressemble » [resãb], « exemple » [czãp], « règle » [reg] et « oncle » [0̃k].

Mais en cas de prononciation de la voyelle finale graphique (15a, c, d) ou d'enchainement (16), les groupes [Cr] et [Cl] en fin de mots conservent leur intégrité :
a. [onorablœ / medamzemesцø]
honorables, mesdames et messieurs
b. [onorabzẽ vite]
honorab' z'invités
c. [utrœleprezidã]
outre les présidents
d. [ləminisrœa $\left.\int \mathrm{i}\right]$
le minisre Achi

\author{
« honorables, mesdames et \\ messieurs » \\ « honorables invités » \\ « outre les présidents » \\ « le ministre Achi »
}




\section{e. [ləpremjeminis] le Prémier minis' \\ [lotrareysisavi] l'autre a réussi sa vie}

« le Premier ministre »

«l'autre a réussi sa vie»

Le groupe [bl] de « honorable » est effectivement prononcé en (15a) [onorablœ] où le «e » muet final est réalisé [œ] $]^{6}$. Mais, sans une voyelle qui le suit, le groupe est réduit; d'où la prononciation [onorab] en (15b). Il en va de même en (15c) et (15d) où, en raison de la prononciation $\mathrm{du}$ « e » final graphique, le groupe [tr] est préservé ici dans " outre » prononcé [utrœ], et «ministre » articulé [minisrœ]. L'exemple (15d) avec « ministre » [minisrœ] laisse à penser que la prononciation de la voyelle finale graphique protège uniquement la consonne finale, car, comme on peut le constater, l'occlusive [t] n'est pas prononcée. On peut même avancer, en observant "ministre » [minis] en (15e), qu'en l'absence d'une voyelle protectrice, le groupe [tr] tombe lorsqu'il est précédé de la fricative [s].

L'exemple (16) montre que l'enchainement protège le groupe $[\mathrm{Cr}]$ de la réduction. En ce cas, la voyelle à laquelle le groupe se lie (ici [a]) se comporte comme une voyelle protectrice (à l'image de [œ] en (15a)). L'exemple (15e) semble confirmer que la prononciation de la voyelle finale graphique protège seulement la consonne finale du groupe, c'est-à-dire [r], et pas le groupe tout entier.

Les suites consonantiques [td], [tn], [kt], [st] font aussi l'objet de réduction. Quand cela se produit, c'est la première consonne de la suite qui tombe. Si l'on prend le cas des mots

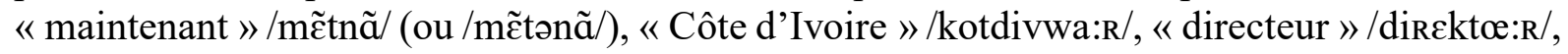
on remarque que les occlusives apico-dentale [t] et dorso-vélaire [k] tombent. Pour ces trois

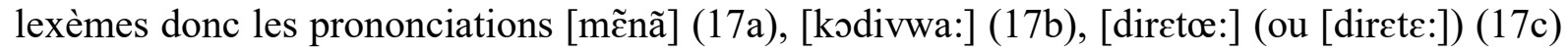
sont les plus fréquentes :
a. [pukwaticlabamẽnã]
pouquoi ti es là-bas mainnant
b. [lavenmãdynkədivwadəpe]
l'avènement d'une Cô d'Ivoi de paix
c. [mwãzєete $\varepsilon \varepsilon f d ə s \varepsilon:$ rvis / sudirete:]
mouan j'ai été chef de service, sous-dirètè

On observe le même phénomène dans « exemple »/Egzãpl/, « texte »/tzkst/, « complexe »

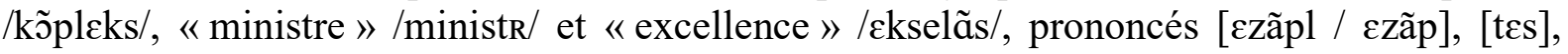

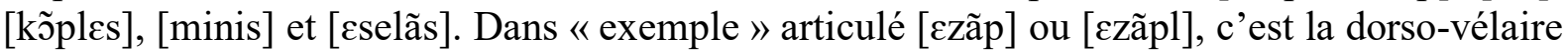
[g] qui est tombée. La réduction de la suite consonantique [st] peut s'observer dans l'articulation de «ministre »/ministr/, " congressiste »/kõgResist/, " houphouëtiste »/ufwetist/, qui deviennent respectivement [minis] (18a), [kõgresis] (18b), [ufwetis] (18c) :

(18) a. [lœkamaradvõalekolpudeveni:minis]
b. [alunanimitedekõgresis]
c. [rasãbləmãdczufwetis]

\section{Leurs camarades vont à l'école pou dévéni minis'}

à l'unanimité des
congressis'
rassemblement des
houphouëtis'

« leurs camarades vont à l'école pour devenir ministres »

$$
\begin{aligned}
& \text { «à l'unanimité des } \\
& \text { congressistes » } \\
& \text { « rassemblement des } \\
& \text { houphouëtistes » }
\end{aligned}
$$

La suite [st] semble se différencier des autres. En cas de réduction, contrairement aux autres suites, nous remarquons que c'est la deuxième consonne, à savoir [t], qui tombe.

\footnotetext{
${ }^{6}$ La voyelle finale prononcée sonne comme celle qu'on peut entendre dans la réalisation du mot « cœur »/kœ: $/$.
} 
La non-réalisation de certaines autres consonnes met en jeu un mécanisme un peu plus complexe. Dans l'articulation de certains mots, les fricatives [3], [v], [s] ne sont pas réalisées, et l'on peut percevoir un bruit de souffle qui donne à penser que ces consonnes sont remplacées par un « h » soufflé. Analysons le cas de [3] à partir des exemples de (19) :
(19) a. [sclãhãkifctu]
c'est l'anhent qui fait tout
«c'est l'argent qui fait tout »
b. [lomnepaalezik
l'homme n'est pas allègique à
«l'homme n'est pas allergique à amãhevitamine] manher vitaminé (manger) ce qui est vitaminé »
c. [nusikwa / isõtrodãhere]
noussis quoi! Ils sont trop danhéré
« les noussis, ils sont trop dangereux »

Il y a altération des lexèmes « argent » /aRzãa/, « manger »/mãze/ et « dangereux »/dãzərø/ qui deviennent « anhent » [ãhã] (19a), « manher » [mãhe] (19b), " danhéré » [dãhere] (19c).

Le phénomène s'observe avec $[\mathrm{v}]$ dans les énoncés suivants :
a. [sahaale]
b. [зəmãhedəneagbagbo]
ça ha aller
je m'en hais donner à Gbagbo
c. [vumahepaekute] vous m'ahez pas écouté
d. [jøhøprãmõlãhãubjẽ]
ye heux prend mon l'anhent, ou bien!

Les différents allomorphes du verbe «aller » sont réalisés avec un « $\mathrm{h}$ » aspiré à la place du « v ». Ainsi, « va »/va/ est prononcé [ha] dans « ça va aller » (20a), « vais » s'articule [he] dans « je m'en vais donner à Gbagbo » (20b). La forme «avez» (de « avoir ») devient [ahe] dans « vous m'avez pas écouté » (20c). La conjugaison du verbe "vouloir » aux trois personnes du singulier donne à observer la même modification. C'est pourquoi «veux »/vø/ en (20d) s'articule [hø].

Le remplacement de $[\mathrm{s}]$ par [h] se remarque dans la prononciation des numéraux allant de « soixante » à « soixante-dix-neuf » inclus. En (21) ci-dessous, pour « soixante-quinze », le participant prononce [swãhãkẽz] au lieu/swasãtkẽz/ :

(21) [salaavãseteswãhãkẽzswãhãkẽz / mẽnãtianupaledekõbjẽ] ça là, avant c'était swanhan-quinze swanhanquinze $^{7}$. Mainnant ti as nous paler de combien?
« ça, avant, c'était soixantequinze (francs CFA l'unité). Maintenant, tu vas nous parler de combien?»

Les occlusives [k], [g], [t] et [n] sont, elles aussi, concernées par le remplacement par [h]. Examinons les exemples suivants :

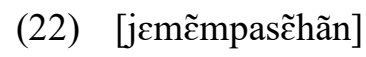

(23) a. [õnєvenirehaderybi]

b. [rehadfamãsz̃tdabobo]

(24) [pehise]

(25) [ग̃nagãhe] y'ai même pas cinhanne on est véni réhader Rubi

réhade femme enceinte d'Abobo

péhi sè on a ganhé
« je n'ai même pas cinquante»

« on est venu regarder Rubi »

« regarde une femme enceinte d'Abobo »

«petite sœur (jeune fille)» « on a gagné »

${ }^{7}$ Au sujet de la réduplication des unités lexicales, on pourra se reporter, par exemple, à Assémou (2018). 
Dans la prononciation du numéral « cinquante » en (22), le participant substitue le $[\mathrm{h}]$ au $[\mathrm{k}]$.

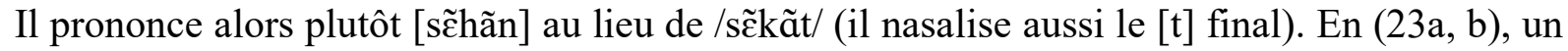
autre participant remplace le [g] par [h] dans «regarder », et prononce [rehade] au lieu de /Rəgan:de/. L'adjectif « petit » et le verbe « gagner », respectivement prononcés [pehi] en (24) et [gãhe] en (25), illustrent le remplacement de [t] et [n] par [h].

La semi-consonne [j] également subit la même modification dans plusieurs mots comme « moyen » (26a), « payer » (26b), « employé » $(26 \mathrm{c})$, « meilleur » (26d, e), " voyez » (26f) :

(26) a.[1вmwahẽdədevlopelakədivwa:]

b. [scləvчølakivapehe]

c. [paleasõnãplwahe]

d. [isว̃padevənylkmøhœ:rzami]

e. [3əsuimehعklijãdeusman]

f. [vuvwaheozoduize $\left.\int \varepsilon z n \tilde{]}\right]$ les moihens de développer la Cô'd'Ivoii

c'est le vieux-là qui va péher

paler à son emploihé

i sont pas dévenus les meuheurs amis?

je suis méhè client dé Ousmane

vous voihez aujourd'hui j'ai chaise non!
« les moyens de développer la Côte d'Ivoire »

« c'est le vieux qui va payer »

« parler à son employé » « ne sont-ils pas devenus les meilleurs amis?»

« je suis le meilleur client de Ousmane»

" vous voyez, aujourd'hui j'ai une chaise, n'est-ce pas?»

Dans des mots comme «moyens»/mwajẽ/, " payer»/peje/, « employé »/ãplwaje/, «meilleur »/mejœ:R/ ou /mejœ:R/, et «voyez»/vwaje/, le [h] aspiré remplace [j], forme phonétique de «y $»$.

Dans la prononciation de « comme ça » et « parce que », on remarque la même substitution. Dans l'articulation de la locution conjonctive "parce que»/parskə/, à l'exception de la consonne initiale [p], toutes les autres sont remplacées par un [h] aspiré, ce qui donne la prononciation [pahe] illustrée par les exemples ci-dessous :

$$
\begin{array}{ll}
\text { a.[scpapahesedu] } & \text { c'est pas pahé c'est doux } \\
\text { c.[scpahejapatravajopei] } & \begin{array}{l}
\text { c'est pahé y a pas travail } \\
\text { au pays }
\end{array}
\end{array}
$$

« ce n'est pas parce que c'est doux (intéressant)»

« c'est parce qu'il n'y a pas de travail au pays »

Les modifications qui surviennent dans la prononciation de la locution adverbiale « comme ça »/komsa/ sont quasi-similaires à celles observées dans la prononciation de «parce que ». Ici également, les locuteurs ne conservent que la consonne initiale. Le $[\mathrm{m}]$ final de $[\mathrm{kom}]$ et le $[\mathrm{s}]$ initial de [sa] sont effacés. À la place, on a l'apparition d'un [h] aspiré, ce qui donne pour « comme ça » la prononciation [kõhã] (avec nasalisation de la voyelle finale [a]) dans les exemples de (28) :

(28) a. [scpaks̃hãfamsekõpst]

b. [sĩ̃vikõhãsepapahesedu] c'est pas conhan femme sé compote si on vit conhan c'est pas pahé c'est doux
« ce n'est pas comme ça qu' une femme se comporte »

« si on vit comme ça, ce n'est pas parce que c'est doux (intéressant) »

\section{Fonctionnement des voyelles}

Les phénomènes de labialisation, de délabialisation, d'amuïssements, et de nasalisation sont très fréquents dans l'articulation des sons vocaliques. 


\section{Les délabialisations et les labialisations}

La labialisation concerne les antérieures étirées /i ; e ; $\varepsilon /$, tandis que les antérieures arrondies $/ y ; \propto ; ø /$ et la centrale arrondie [ə] font l'objet de délabialisation. Ces modifications ne sont pas aléatoires. Les faits montrent que chaque voyelle est remplacée par celle qui s'oppose à elle du point du vue de la labialité. Ainsi, chaque étirée est remplacée par l'arrondie correspondante, et vice versa. Les faits montrent également que les deux phénomènes obéissent à certaines contraintes. Analysons les exemples de (29):
(29)
a. [ticrasazjeal`typa:1]
b. [tipezamẽefasesa]
c. [tiscpapukwamẽilela]

ti es rassasié alo tu paales « tu es rassasié, alors tu parles » ti pé jamain effacer ça «tu ne peux jamais effacer cela» ti sais pas pouquoi main il « tu ne sais pas pourquoi, mais il est est là

En (29a), le locuteur alterne [i] et [y] dans la prononciation du pronom «tu ». Dans « tu es rassasié », le 'u' de «tu » devient ' $i$ '. Dans «alors tu parles », par contre, le ' $u$ ' de « tu » est réalisé normalement. L'énoncé (29b) correspond, en français standard, à « Tu (ne) peux jamais effacer ça »/typøzameefasesa/. Ici, le locuteur délabialise les voyelles /y/ et /ø/ qui deviennent [i] et [e], leurs correspondantes antérieures étirées respectives. Ces alternances sont fréquentes. Ainsi, la réalisation du schwa [ə] dans «venir »/vəni:R/ et « devenir »/dəvəni:R/, pour ne prendre que ces exemples, est fonction de la voyelle de la syllabe subséquente. Au participe passé, en raison du 'u' /y/ qui apparait dans les formes « venu »/vəny/ et « devenu »/dəvəny/, le schwa [ə] est réalisé normalement. Mais, à l'infinitif, il n'est pas rare que cette voyelle soit remplacée par la voyelle [e], l'antérieure étirée qui lui est le plus proche.

À l'inverse, les voyelles antérieures étirées sont labialisées dans l'environnement des antérieures arrondies correspondantes :

(30) a. [omyluøદvametsõfamøadzovã]

b. [عtrepridəpєkõtyny]

c. [3єrøynilemãbdəmว̃pati]

d. [ləvisprezidãdəlarøpyblik]

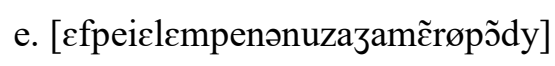

f. [isว̃padevənylemøhœ:rzami]

g. [ləvœ̃nœfdesãbr]

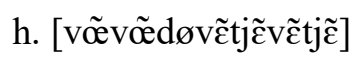

au mulueu è va mett' son fameux adjovan

être épris de paix contunue

j'ai reuni les memb' de mon pati

le vice-président de la republique

FPI-LMP ne nous a jamain repondu

$i$ sont pas devenus les meuheurs amis?

le vungt-neuf décembre

vungt vungt-deux vingtien vingtien
« au milieu elle va mettre son fameux adjovan»

« être épris de paix continue »

«j'ai réuni les membres de mon parti »

«le vice-président de la république »

« le FPI-LMP ne nous a jamais répondu »

« ne sont-ils pas devenus les meilleurs amis ?»

« le vingt-neuf décembre »

« vingt vingt-deux vingt-etun vingt-et-un »

On voit ici que les voyelles antérieures étirées sont remplacées par leurs correspondantes arrondies. En (30a) et (30b) la voyelle /i/ est remplacée par [y] dans « milieu » [myluø] et « continue » [kõtyny]. Dans les énoncés $(30 \mathrm{c}, \mathrm{d}, \mathrm{e}),[\varnothing]$ se substitue à [e] dans « réuni » [røyni], « république » [røpyblik], « répondu » [røpõdy]. Le remplacement de /e/ par sa correspondante arrondie [ø] s'observe avec «meilleurs » qui, en (30f), est prononcé [møhœ:r] au lieu de

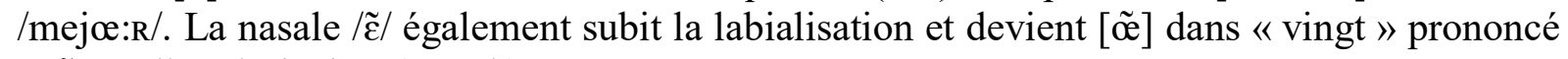

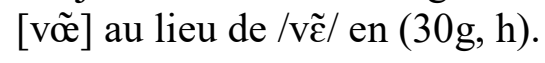


D’une manière générale, la prononciation des antérieures arrondies pose quelques problèmes. Deux cas de figure se présentent: soit elles sont remplacées par leurs correspondantes étirées (ou la voyelle la plus proche, dans le cas du schwa) soit elles assimilent la (les) voyelle(s) étirée(s) qui les précèdent. Il y a délabialisation dans le premier cas, et labialisation dans le deuxième. On pourrait y voir une harmonie d'arrondissement.

\section{Les amuïssements}

L'amuïssement est un processus qui aboutit à la non prononciation d'un son qui (normalement ou autrefois) était articulé. On assiste à ce phénomène dans la prononciation de " président »/prezidã/, « professeur »/profesœ:R/ et « appétit»/apeti/. La voyelle [i] est amuïe dans « président », et [e] n’est pas prononcé dans « professeur » et « appétit »:
(31) a. [ləprezdãufwebwani]
"le prézdent Houphouët-
Boigny»
« le président
Houphouët-Boigny »
b. [ləprezdãdəlarepyblikapale]
" le prézdent de la république a palé »
« le président de la
république a parlé »
c. [profsœdədrwa]
"Profseur de droit »
«professeur de droit »
d. [bõnapti]
«Bon ap'tit!"
«bon appétit!»

Dans la prononciation de «professeur » chez certains locuteurs, ce n'est pas seulement la voyelle [e] contenue dans la forme phonique /profesœ:R/ qui est amuïe, mais toute la syllabe [fe-] dont cette voyelle est le noyau. Cela aboutit à la prononciation [proosœ:].

\section{Les nasalisations}

Les voyelles orales $[\varepsilon]$ et [a] sont nasalisées dans les mots « mais » $/ \mathrm{m} \varepsilon /$, « même »/mem/,

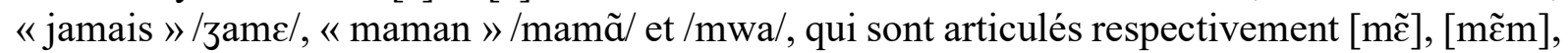

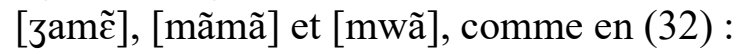

(32) a. [mẽkeskilvanuzãvwajemẽtnã]

b. [õnupa:Imalmẽõsãgabadesa]

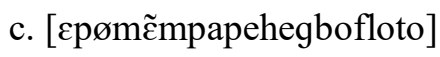

d. [fozamz̃təfedenemi]

e. [regadmamitmãmãprãpuprepare]

f. [gbagboemwãõnapyctenemi] main qu'est-ce qu'il va nous envoyer maintenant

on nous paale mal main on s'en gaba dé ça

è peut maimme pas péher gbofloto

faut jamain te fai d'ennémis

régade mamite mamman prend pour préparer

Gbagbo et mouan on a pu êt' ennémis
« mais qu'est-ce qu'il va nous envoyer maintenant »

« on nous parle mal mais on s'en fout»

« elle ne peut même pas payer gbofloto »

« il ne faut jamais te faire d'ennemis »

« regarde la marmite que maman utilise pour cuisiner »

« Gbagbo et moi, on a pu être ennemis »

Il semble, cependant, que le phénomène soit circonscrit à quelques mots bien précis, notamment ceux présentés ici. 


\section{L'opposition [o] / [0]}

Les voyelles [o] et [0] s'opposent par leurs degrés d'aperture. En effet, même si dans certaines présentations elles sont classifiées comme des voyelles moyennes, il faut savoir que [o] est mi-fermée et que [o] est mi-ouverte. Sur le plan de leur position dans la syllabe (ou distribution syllabique), ces deux voyelles s'excluent mutuellement. On dit qu'elles sont en distribution complémentaire, et la loi qui régit leur distribution est appelée loi de distribution complémentaire ou loi de position. En français standard, selon la loi de position, [o] apparait en syllabe accentuée ouverte, et [0] en syllabe accentuée fermée (Léon et Léon, 1997 : 33). D'après les données que nous avons analysées, cette loi semble ne pas toujours opérer en français de Côte d'Ivoire. Des réalisations telles que celles de (33) ci-dessous montrent en effet que [0] peut apparaitre aussi bien en syllabe fermée qu'en syllabe ouverte.
(33) a. [sakderiegbolo]
sac dé riz est gbôlô
« le sac de riz est vide»
b. [devlopelakodivwa:]
développer la Cô'd'Ivoii
« développer la Côte d'Ivoire»
c. [ivatapepoto]
i va taper poteau
« il va échouer»

Dans [gbo-lo] (33a), la voyelle [0] apparait en syllabe ouverte, là où, en français standard, la loi de distribution complémentaire prévoit sa correspondante fermée [o]. Par ailleurs, même si dans [ko-divwa:] (33b) [o] apparait en syllabe ouverte, la réalité est que nous sommes bien en face d'une syllabe fermée ; la prononciation exacte étant/kot-divwa:R/.

Notons que le français admet quelques exceptions à la loi de position (Léon et Léon, 1997 : 34). Dans les mots français présentant un "o" avec un accent circonflexe au niveau graphique, cette voyelle se prononce [o] en syllabe fermée. C'est pourquoi un mot comme "côte » se réalise [kot]. Cette règle non plus n'est pas respectée, et dans les mots français présentant un "o" avec accent circonflexe, le "ô" est généralement prononcée [o]. On peut même évoquer un problème d'orthoépie. En effet, sur le plan graphique, un mot d'origine ivoirienne comme " gbôlô » s'écrit (dans la presse écrite par exemple) avec un accent circonflexe sur chaque "o" pour indiquer justement qu'ici c'est le "o" mi-ouvert [o] qui est de rigueur. C'est d'ailleurs cette manière d'écrire que nous avons adoptée ici. Cette règle d'écriture peut cependant dérouter les autres francophones (les natifs notamment) dans la mesure où elle indique le contraire de ce que prescrit le français standard.

On observe tout de même que la loi de position est respectée en (33c) dans [po-to]. Mais, alors que [०] peut apparaitre en syllabe fermée et en syllabe ouverte, tout semble indiquer que [o] est exclu en syllabe fermée. En effet, un mot comme " côte »/kot/ est prononcé [kot] par les participants. Le mot « Côte d'Ivoire » par exemple reçoit deux prononciations. Dans l'une, le « $\mathrm{t} »$ de « côte » n'est pas réalisé, ce qui conduit à la prononciation [kodivwa:]. Dans l'autre, cette consonne est effectivement réalisée, et l'on obtient la prononciation [kotdivwa:]. Mais, comme on peut le voir, dans un cas comme dans l'autre c'est la voyelle [o] qui apparait. Ces deux prononciations posent problème. La première n'est pas conforme à l'énoncé général de la loi de position. La seconde par contre ne respecte pas l'exception que cette loi prévoit à propos de la prononciation $\mathrm{du} \ll \mathrm{o} »$ avec accent circonflexe.

À l'analyse des données, un autre fait concernant l'opposition [o] / [0] apparait. Considérons le corpus suivant :
(34) a. [sakderiegbolo]
sac dé riz est gbôlô
« le sac de riz est gbôlô (vide)»
b. [pehegbofloto]
péher gbofloto
«payer gbofloto»
c. [scleksko]
c'est les côcô
« ce sont les côcô (quémandeurs)»

En observant les mots " gbôlô » [gbolo], « gbofloto » [gbofloto], et « côcô » [koko], on peut remarquer que les voyelles d'un même mot ont le même timbre. Il n'y a pas d'alternance [o] / 
[0] au sein d'un même mot. Ainsi, dans une structure polysyllabique de type CV1CV2 (où C symbolise une consonne, et V une voyelle), V1 et V2 correspondent à la même voyelle. On pourrait y voir une tendance à l'harmonie.

\section{Discussion}

Ce travail, qui s'intéresse particulièrement au fonctionnement des sons du français parlé en Côte d'Ivoire, vise à expliquer comment les particularités phonétiques de ce français éclairent la dynamique de la langue française dans ce pays. Pour ce faire, nous avons procédé à un inventaire des sons avant d'en aborder le fonctionnement.

L'inventaire consonantique montre un système plus étendu dont la nouveauté réside dans la présence de six (6) consonnes non attestées en français standard. Il s'agit des occlusives labiovélaires [kp] et [gb], des affriquées [t] et [d]], de l'occlusive palatale [f], et de la consonne alvéolaire battue $[r]$. L'inventaire des voyelles révèle l'absence du [a] postérieur et de sa nasale [ã] dans le français parlé. Ces résultats montrent un système phonétique différent de celui du français standard.

Le fonctionnement des unités phoniques également présente des particularités. Dans le fonctionnement des consonnes, on note des chutes en position finale, des cas de réduction de certains groupes et suites consonantiques, ainsi que des aspirations. Le fonctionnement des voyelles est, quant à lui, marqué par des labialisations et délabialisations, des nasalisations, des amuïssements, ainsi que le non-respect de la loi de position qui régit la distribution syllabique des voyelles [o] et [0].

Si quelques-unes des particularités soutiennent la thèse de l'influence des langues maternelles ivoiriennes, la grande majorité des faits observés s'explique plutôt par des facteurs plus divers, qui ne sont pas forcément en lien avec ces langues.

\section{Langues maternelles ivoiriennes et particularismes phonétiques}

On ne peut pas affirmer que les langues maternelles ivoiriennes ne sont pas en cause dans les particularismes phonétiques observés. Le fait est que ces langues impriment leurs marques dans le français oral, notamment à travers les emprunts lexicaux et les habitudes articulatoires des locuteurs.

\section{Le rôle des emprunts lexicaux}

On peut rendre compte de la présence des occlusives labio-vélaires [kp] et [gb], des affriquées [t] et [d]], et de l'occlusive palatale [f] dans l'inventaire par les emprunts lexicaux $\mathrm{du}$ français aux langues maternelles ivoiriennes. Dans la situation de contact de langues qui caractérise la Côte d'Ivoire, le français côtoie quotidiennement, en effet, une diversité de langues maternelles autochtones dont les systèmes phonologiques comportent les labio-vélaires [kp] et [gb] (Charette, 1984 ; Kaboré et Tchagbalé, 1998 ; Kouadio et Kouamé, 2004 ; Zogbo, 2005 ; Vogler, 2017 ; Ahoua et Kouadio, 2017 ; Adopo, s.d. ; entre autres). Or il se trouve que certains mots de ces langues, qui comportent les consonnes susmentionnées, sont passés dans le vocabulaire du français ivoirien. Aussi semble-t-il normal que ces consonnes intègrent aussi l'inventaire des sons du français parlé dans ce pays. Toutefois, pour ce qui est du cas spécifique des affriquées [t] et [d] , il serait difficile d'expliquer leur présence dans l'inventaire par des emprunts directs aux langues maternelles ivoiriennes. Il faudrait plutôt les mettre en rapport avec la palatale $[\mathrm{f}]$.

Les affriquées [d] et [t] fonctionnent respectivement comme substituts de l'occlusive palatale sonore [f] et de sa correspondante sourde [c] qu'on retrouve dans les langues ivoiriennes, comme l'attestent également les travaux des auteurs ci-dessus cités. Certains mots 
non-attestés en français standard mais qui font désormais partie du vocabulaire du français parlé en Côte d'Ivoire tels que "adjovan » et "attiéké » sont prononcés respectivement [àjóvá] et [àcèké] dans les langues d'emprunt. Ce faisant, ce que les francophones ivoiriens prononcent [dङ] dans un mot comme « adjovan » est en réalité un [f], c'est-à-dire un son semblable à ce que l'on peut entendre à l'initial du mot français « guirlande » par exemple. Cela explique pourquoi nous avons deux variantes lexicales, à savoir [adzovã] et [ajovã], qui désignent la même réalité. Il en va de même du mot " attiéké » qui, dans les langues d'emprunt, se prononce [àcèké] (avec quelques différences tonales légères en fonction des groupes linguistiques). La présence de ces deux affriquées dans le système phonétique résulte donc d'un emprunt aux langues maternelles ivoiriennes, mais avec une réinterprétation des consonnes occlusives palatales d'origine, sans doute par préférence des locuteurs pour les affriquées. Il arrive même que ces occlusives palatales et les affriquées soient remplacées par des formes syllabiques composées d'une consonne alvéo-dentale ([d] ou [t]) et de la voyelle palatale [i]. [t] et [c] deviennent [ti] tandis que [f] et [d]] deviennent [di]. C'est ce qui explique que pour un mot comme "attiéké » on a les prononciations [atfeke] et [atjeke], la deuxième prononciation correspondant à la substitution de la syllabe [ti] à [c] ou [t]]. Cette substitution est fréquente dans de nombreux mots empruntés aux langues maternelles ivoiriennes, y compris des noms propres de personnes ou de lieux.

La présence des nasales [ĩ] et [ũ] dans l'inventaire vocalique s'explique également par les emprunts lexicaux du français aux langues maternelles ivoiriennes, suivant le schéma décrit pour les consonnes. Comme on peut le remarquer en observant les données, la nasalisation des voyelles [i] et [u] n'est pas systématique dans la prononciation des mots français. Si ces voyelles entrent dans l'inventaire, c'est parce que certains mots inconnus en français standard sont entrés dans le vocabulaire du français parlé en Côte d'Ivoire. Ces mots nouveaux sont pour la plupart d'origine nouchi, le parler jeune ivoirien qui, lui-même, emprunte aux langues maternelles ivoiriennes, ou crée des mots nouveaux. La variété nouchi est réputée pour son vocabulaire très dynamique. Les mots qui deviennent trop communs finissent par intégrer le vocabulaire populaire. C'est le cas de «gbonhin » [gbõhĩ] et «tchoun » [tũu], qui ont perdu leur caractère ésotérique pour devenir vulgaires. Le nouchi contribue ainsi à l'enrichissement lexical du français parlé en Côte d'Ivoire, ce qui peut expliquer l'extension du système phonologique en général. Rappelons aussi que les nasales [ĩ] et [ũ] font partie des systèmes des langues maternelles ivoiriennes (Kouadio et Kouamé, 2004 ; Adopo, s.d.).

Il apparait ainsi que les emprunts lexicaux ne conduisent pas uniquement à un enrichissement lexical du français. Il y a également modification du système phonologique de cette langue.

\section{Les habitudes articulatoires des locuteurs et les conditions d'apprentissage du français}

Certaines des particularités mises en lumière par l'inventaire ne tiennent pas de l'emprunt lexical. Elles semblent plutôt mettre en avant les habitudes articulatoires des locuteurs, ainsi que les conditions d'apprentissage du français. C'est le cas de la consonne battue [r]. Comme le montrent les données, elle peut apparaitre dans des mots du français standard. Nous pensons qu'il s'agit d'une réinterprétation du « $\mathrm{R}$ » français par les locuteurs qui ne parviennent pas à réaliser cette consonne, et qui ont recours à leurs langues maternelles pour y trouver un son qui lui est proche. Dans le cas qui nous concerne, la participante qui prononce cette variante de « $\mathrm{R} »$ est d'origine baoulé, une langue kwa qui n'a pas le « $\mathrm{R} »$ français mais un « $\mathrm{r}$ » qui a la particularité d'être «"battu", plus proche du $l$ que du $r$ français » (Kouadio et Kouamé, 2004 : 18). La réinterprétation du « $\mathrm{R} »$ français chez cette participante peut s'expliquer par le fait qu'il s'agit d'une personne d'un certain âge, qui n'a certainement pas appris le français en contexte formel. On peut donc avancer qu'elle n'a pas appris à réaliser le « $\mathrm{R}$ » français au cours de son enfance, d'où la difficulté. Les thèses psychologiques sur l'apprentissage des langues définissent en effet un âge optimum et un âge critique au-delà desquels l'apprentissage des 
langues devient difficile (Azzam-Annachi, 2005). On peut ajouter que les conditions d'apprentissage du français (contexte informel, et sans doute tardif) ne lui ont pas permis d'apprendre la consonne française « $\mathrm{R} »$, d'où la réalisation $\mathrm{du}$ « $\mathrm{r} »$ battu (attesté dans sa langue maternelle) en lieu et place du « $\mathrm{R} »$ français. Cela signifie que cette réinterprétation ne s'étend pas à tous les locuteurs issus du même groupe linguistique que cette participante. En d'autres termes, on ne peut pas affirmer que tous les Baoulé prononcent systématiquement le « $\mathrm{R} »$ battu à la place $\mathrm{du}$ « $\mathrm{R} »$ français.

L'inventaire des voyelles révèle l'absence du [a] postérieur dans le français parlé. Cette particularité également relevée par Boutin et Turcsan (2009), ne peut toutefois s'expliquer par la (quasi) disparition de cette voyelle en français moderne. Il faudrait plutôt convoquer les bases articulatoires des langues maternelles ivoiriennes. En effet, la voyelle postérieure orale [a] et la nasale correspondante [ã ] ne sont pas attestées dans les langues maternelles ivoiriennes. Aussi les locuteurs n'ont-ils pas pu être en contact avec ces sons dans leur enfance. Or nous savons que, même si l'enfant est capable de produire tous les sons vocaux possibles au cours de ses deux premières années de vie, ses «productions spontanées vont décroitre progressivement jusqu'à que [sic] l'enfant ne retienne plus que les sons fournis par son entourage » (H. RicaudDroisy et al., $2009:$ 73). Cela démontre que «l'enfant construit sa langue à partir des formes orales qu'il entend » (Aimard, $1996: 71$ ). On comprend donc que les Ivoiriens qui sont nés en France notamment puissent réaliser ces sons, mais pas ceux qui sont nés et ont grandi en Côte d'Ivoire. Pour ces derniers, seule l'école pouvait leur permettre d'apprendre ces sons. Or, même à l'école, le «A $\mathrm{A}$ » postérieur et sa nasale sont remplacés par les voyelles qui leurs sont proches et qui existent dans les langues maternelles ivoiriennes, à savoir [a] et [ã]. Des échanges que nous avons eus avec un professeur de $\mathrm{CAFOP}^{8}$ (ancien maitre d'école primaire) et un Chef de circonscription de l'enseignement préscolaire et primaire, il ressort en effet que la différence entre les deux « A » français n'est pas enseignée aux élèves pendant les cours de français. Aussi, étant donné que dans l'apprentissage du français, les bases sont posées au préscolaire et au primaire, il nous semble clair que si dans les classes de ces cycles les enfants n'apprennent pas à réaliser $[\mathrm{a}]$ et $[\tilde{a}]$ et à les distinguer de leurs correspondantes antérieures [a] et [ã], ceux-ci ne pourront que réaliser les antérieures (qui existent dans leurs langues) en lieu et place des postérieures. De plus, la différence entre les antérieures et leurs correspondantes postérieures étant ténue, la distinction est quelque peu difficile. Cela facilite par ailleurs la substitution des premières aux secondes. C'est donc aussi un cas de réinterprétation qui trouve son origine à la fois dans les habitudes articulatoires que les locuteurs ont héritées de leurs langues maternelles, et dans les conditions d'apprentissage du français.

Dans ces deux cas de réinterprétation, les locuteurs recourent à leurs langues maternelles parce que leurs habitudes articulatoires ne leur permettent pas de réaliser certains sons du français. Ce faisant, à la place des unités que leurs répertoires phoniques ne contiennent pas, ils emploient d'autres unités plus ou moins proches, que leur fournissent leurs langues maternelles. Les origines de ces réinterprétations sont donc lointaines. Elles semblent remonter à l'enfance, pendant la phase d'acquisition/apprentissage des langues.

En définitive, nous avons lieu de dire que ce sont les emprunts lexicaux aux langues autochtones et les réinterprétations qui sous-tendent l'extension des systèmes consonantique et vocalique du français parlé. Par ailleurs, les bases articulatoires des langues maternelles des locuteurs peuvent justifier l'absence dans l'inventaire de certains sons vocaliques français.

\footnotetext{
${ }^{8}$ Centre d'animation et de formation pédagogique. C'est l'institution chargée de la formation des enseignants du primaire en Côte d'Ivoire.
} 


\section{Le jeu des principe et lois phonétiques}

Si l'inventaire des sons rappelle l'importance de la situation sociolinguistique ivoirienne dans la particularisation du français parlé en Côte d'Ivoire, leur fonctionnement par contre révèle le jeu de certains principes et lois phonétiques.

Boutin et Turcsan (2009) ont remarqué que la finale syllabique en français de Côte d'Ivoire constitue une position faible dans laquelle des consonnes, notamment les liquides [1] et [r], s'affaiblissent et tombent. Notre étude confirme cette observation. Toutefois, il est à noter que ceci n'est pas une spécificité du français parlé en Côte d'Ivoire. Cette particularité (que l'on retrouve dans d'autres variétés du français) peut s'expliquer par le principe de la coarticulation, la loi du moindre effort, et, surtout, la loi des forces consonantiques.

Les langues sont ainsi faites que lorsqu'une personne parle, elle n'articule pas chaque son séparément; chaque son s'enchainant plutôt avec celui qui le précède et avec celui qui le suit (Léon et Léon, 1997 : 57). Ce phénomène, connu sous le nom de coarticulation, entraine, si l'on parle « spontanément, familièrement et vite (...) beaucoup d'accidents de prononciation ».

Ces «accidents » sont également liés à la loi du moindre effort dont parle Martinet (2008 [1960] : 181) :

L'évolution linguistique est conçue comme régie par l'antinomie permanente entre les besoins communicatifs de l'homme et sa tendance à réduire au minimum son activité mentale et physique. Ici, comme ailleurs, le comportement humain est soumis à la loi du moindre effort selon laquelle l'homme ne se dépense que dans la mesure où il peut atteindre aux buts qu'il s'est fixés.

Cette tendance naturelle de l'homme à ne pas en faire plus qu'il n'en faut quand il s'agit d'atteindre un but donné s'applique aussi au domaine linguistique : il a tendance à faire des économies d'énergie quand il parle. Il s'ensuit qu'il peut articuler moins bien, tant que ce qu'il dit (en faisant des économies) n'entrave pas la réussite de la communication.

Mais, au-delà, il y a aussi que l'articulation des consonnes est soumise à la loi des forces consonantiques (Léon et Léon, 1997 : 59), qui détermine des consonnes fortes (ou faibles) par nature ou de par leur position dans la syllabe. Ainsi, par nature, les occlusives $[p ; t ; k ; b ; d$; g] sont plus fortes que les fricatives $\left[\mathrm{f} ; \mathrm{s} ; \mathrm{z} ; \mathrm{v} ; 3 ; \int ; \mathrm{R} ; \mathrm{l}\right]$; et les consonnes sourdes $[\mathrm{p} ; \mathrm{t}$; $\left.\mathrm{k} ; \int ; \mathrm{f} ; \mathrm{s}\right]$ plus fortes que leurs correspondantes sonores $[\mathrm{b} ; \mathrm{d} ; \mathrm{g} ; 3 ; \mathrm{v} ; \mathrm{z}]$ et les consonnes nasales. Le critère de la position dans la syllabe permet de distinguer deux cas de figure. $\mathrm{Si}$ deux consonnes sont en contact dans une même syllabe, les forces consonantiques sont définies en fonction de la nature des consonnes en contact. Dans le cas où les consonnes en contact appartiennent à deux syllabes distinctes, la consonne en finale syllabique est faible par rapport à celle qui se trouve en début de syllabe. Autrement dit, la fin de syllabe est une position faible par rapport à la position initiale syllabique qui, elle, est une position forte. Le fait est que « la consonne qui termine la syllabe précédente s'appelle implosive, celle qui commence la syllabe suivante est appelée explosive. [Et il se trouve que] l'explosive a toujours plus d'énergie que l'implosive ». En vertu de la loi des forces consonantiques, la consonne forte assimile celle qui est faible.

Comme nous le disions plus haut, ces lois peuvent, indépendamment de toute référence aux habitudes que les locuteurs ont héritées de leurs langues maternelles, permettre de rendre compte des différentes chutes consonantiques observées. Ainsi, on peut expliquer la chute de [1] dans les pronoms personnels « il(s)» et « elle(s)» respectivement articulés [i] et [ $\varepsilon$ ] devant un verbe à initiale consonantique par la loi du moindre effort, mais surtout par la loi des forces consonantiques. Dans ces mots, [1] est en position finale syllabique, une position faible. Cela a pour conséquence une articulation avec peu d'énergie. La liquide étant prononcée faiblement, elle est facilement assimilée par la consonne suivante. Notons que cette prononciation 
particulière de pronoms «il(s)» et «elle(s)»s'observe également chez les Français natifs, « dans la conversation familière, même en public » (Léon et Léon, 1997 : 108).

Il en va de même de la réduction des groupes consonantiques de types «Consonne $+\mathrm{R}$ » $[\mathrm{Cr}]$ et « Consonne $+1 »[\mathrm{Cl}]$, que l'on observe ailleurs, notamment en France, au Canada et en Louisiane (Léon et Léon, 1997 : 104, 107-108 ; Lyche, 2000 : s.p.), et qui répond, elle aussi, à la loi des forces consonantiques. En général, deux consonnes en contact dans un mot se séparent en cas de syllabation. Ce n'est pas le cas de [r] et [1] qui ne se séparent pas de la consonne qui les précèdent. Les groupes $[\mathrm{Cr}]$ et $[\mathrm{Cl}]$ correspondant à des consonnes en contact dans une même syllabe, c'est la nature des consonnes qui régit l'assimilation. Or les liquides [r] et [1] sont plus faibles que les occlusives qui les précèdent, donc elles tombent. Cela permet de comprendre pourquoi un mot comme "cadre » se prononce [kad] et que "honorable » est articulé [onorab]. En plus d'être plus faibles que les occlusives par leur nature, [r] et [1] sont également en finale syllabique, une position implosive. Prononcées avec moins d'énergie, les deux liquides s'affaiblissent et/ou tombent. Dans un mot comme «ministre » prononcé [minis] au lieu de /ministr/, le groupe [Cr] entier tombe. Dans ce cas-ci, la liquide [r] tombe en premier, puis, la fricative $[\mathrm{s}]$ assimile l'occlusive $[\mathrm{t}]$ forte par nature mais faible parce qu'en position implosive. On voit donc qu'une consonne faible par nature (la fricative) peut assimiler une consonne forte par nature mais rendue faible de par sa position (ici l'occlusive $[\mathrm{t}]$ ).

Ces mêmes lois phonétiques s'appliquent aux consonnes en contact mais appartenant à des syllabes différentes. Les mots « Côte d'Ivoire »/kotdivwa:R/ et « maintenant »/mẽtnã/sont une belle illustration de l'application de la loi des forces consonantiques. Dans la prononciation de « Côte d'Ivoire » et «maintenant », bien souvent le [t] n'est pas réalisé ; ce qui aboutit aux prononciations [kodivwa:] et [mẽnã]. Si nous appliquons les principes de la syllabation, "Côte d'Ivoire » sera syllabé /kot-di-vwa:R/ et «maintenant»/mẽt-nã/. Dans les deux cas, la consonne [t] est en position implosive (faible) suivie d'une consonne forte de par sa position initiale syllabique (position explosive). Dans /kot-di-vwa: $\mathrm{R} /$, [t] précède [d] qui est plus forte de par sa position. Dans /mẽt-nã/, [t] précède [n]. Dans les deux cas, [t] est assimilé par la consonne suivante. Il s'agit ici d'une assimilation totale. [t] devient donc [d] dans la prononciation de "Côte d'Ivoire ». Dans l'articulation de «maintenant », [t] devient d'abord sonore, donc [d]. Puis, il y a nasalisation de [d] qui, en fin de compte, devient [n]. On obtient, dans un cas comme dans l'autre, des géminées (deux consonnes identiques) qui, par la suite, sont simplifiées en une seule pendant l'articulation de chacun des mots. Comme le disent Léon et Léon (1997: 60), l'assimilation totale de [t] dans l'articulation de «maintenant » qui devient [mẽnã] dans certains registres du français standard (et en cadien ; Lyche, 2000) est « un bel exemple d'usure de la langue ou d'économie linguistique ».

Le jeu des forces consonantiques et la loi du moindre effort peuvent expliquer les chutes consonantiques dans la prononciation d'autres mots comme " exemple » [Ezãp], « complexe » [kõples], « excellence » [Eselãs], «ministre » [minis]. Le cas du [k] non-réalisé dans « complexe »/kõpleks/ semble beaucoup plus tenir de la loi du moindre effort. Selon Malécot (1977 : 31), « les occlusives vélaires, quel que soit leur mode d'articulation, sont les plus "fortes" de toutes les consonnes ». Autrement dit, leur articulation est celle qui nécessite le plus d'énergie. Cela laisse à penser que la non-réalisation de la vélaire [k] dans des mots comme « complexe» répond plus à un besoin d'économie.

Au-delà des chutes consonantiques, les données que nous avons analysées suggèrent que la prononciation du français parlé en Côte d'Ivoire est aussi marquée par le remplacement de certaines consonnes par un bruit de souffle qui rappelle le $\mathrm{h}$ aspiré. Cette modification connue dans de nombreuses variétés du français et dans d'autres langues du monde correspond à ce que l'on appelle la spirantisation. Dubois et al. (1994:440), la définissent comme « (...) le passage d'un son dont l'articulation comporte une occlusion du chenal buccal à un son dont l'articulation comporte un resserrement du chenal buccal ». La spirantisation consiste donc à 
passer d'une consonne occlusive à une consonne fricative. Mais il y a aussi spirantisation quand une fricative devient un $\mathrm{h}$ aspiré. Dans de nombreux cas, en effet, la consonne résultant de la spirantisation est la spirante laryngale $[\mathrm{h}]$ ou $\mathrm{h}$ aspiré. L'analyse montre que la majorité des cas de spirantisations observées aboutit au remplacement d'une consonne (occlusive ou fricative) par un h aspiré. Notons que le $\mathrm{h}$ aspiré ne fait pas partie de l'inventaire consonantique du français. Sa présence dans cette langue s'explique par les emprunts aux langues germaniques, au grec et au latin. Les mots français commençant par un $\mathrm{h}$ aspiré sont d'origine germanique, tandis que ceux débutant par un $\mathrm{h}$ muet sont d'origine grecque ou latine (Exare, 2017 : 2-45). Il est à noter également que le h aspiré résulte de la lénition (affaiblissement) d'autres consonnes occlusives ou fricatives (Bittner, 1995 ; Léon et Léon, 1997 ; Lafkioui, 2006 ; Exare, 2017). Les consonnes spirantisées que nous avons relevées sont la vélaire [g] comme dans la prononciation de «regarder », la fricative [v] dans certaines formes conjuguées de verbes comme « vouloir », « aller », « avoir », la fricative [3] dans les mots « argent », « manger » et " dangereux » par exemple, la fricative [s] dans la prononciation de « comme ça » et «parce que », la nasale [n] dans « gagner », ainsi que l'occlusive [t] dans l'articulation de «petit(e)». Ces modifications peuvent s'expliquer par une lénition des consonnes. On peut évoquer une assimilation double, dans la mesure où le phénomène s'observe principalement en contexte intervocalique. Si l'on s'appuie sur le cas de «comme ça»/kom-sa/, le [m], en position implosive, s'affaiblit et tombe. Mais on peut admettre que cette consonne nasalise préalablement la voyelle [0], ce qui débouche sur une prononciation qui ressemble à [kõ-sa]. Après la nasalisation de [0] et la chute de [m], la fricative [s] se retrouve en position intervocalique, une position décrite comme favorisant les spirantisations (Bittner, 1995 : 19). Dans cette position, les locuteurs ne réalisent ni l'occlusion ni le resserrement du conduit vocal nécessaires à la production des occlusives et des fricatives. Par négligence (Exare, 2-52) ou par économie, les consonnes sont prononcées avec moins d'énergie. Elles ont ainsi tendance à être réalisées de façon continue, à la manière des voyelles précédentes ou subséquentes. Cette explication peut être appliquée à tous les cas de spirantisation, y compris les cas où la consonne aspirée est en initiale syllabique. Dans ces cas, c'est la coarticulation qui crée le contexte intervocalique favorisant la spirantisation. Dans « je veux... » prononcé [jəhø] par exemple, on peut observer que la fricative [v] à l'initiale de «veux»/vø/ se retrouve en position intervocalique dans le groupe rythmique / zəvø/. Par ailleurs, une fricative pouvant être convertie en semi-consonne selon le schéma "occlusive $\Rightarrow$ fricative $\Rightarrow$ semi-voyelle ( $\Rightarrow$ voyelle) » (Lafkioui, 2006), on peut parler de spirantisation quand [3] devient [j] dans la prononciation du pronom «je» [jə/je]. Notons que la position initiale favorise aussi les aspirations (Bittner, 1995 : 19). On retrouve aussi la spirantisation de [3] qui devient [h] dans certaines variétés du français, y compris en France (Léon et Léon, 1997 ; Bittner, 1995 ; Exare, 2017). À ce propos, Léon et Léon (1997 : 104) rapportent :

Spirantisation de CH et $J$, qui deviennent H. En Acadie et en Beauce québécoise, on entend, comme en Charentes dans le Poitou en France, que CH et J sont devenus des $H$ aspirés. J'ai une chemise est prononcé [Heynhmiz]. [H] est un souffle voisé de [h] qui est un souffle non voisé.

Pour ce qui est des voyelles également, des lois phonétiques peuvent permettre d'expliquer les particularités qui apparaissent dans leur fonctionnement. Il semble que l'alternance de $i$ et $u$ dans la prononciation du pronom personnel «tu » ou encore la labialisation du $i$ de « continu » sont à mettre en rapport avec une harmonie vocalique dans laquelle non seulement [y] et [i], mais aussi $[\mathrm{e} ; \varepsilon ; \varnothing ; \propto ; ə ; \tilde{\varepsilon} ; \tilde{\propto}]$ sont impliqués. Même si l'harmonie vocalique est connue dans les langues maternelles ivoiriennes (Kaboré et Tchagbalé, 1998 ; Kouadio et Kouamé, 2004 ; Zogbo, 2005), cela ne peut justifier l'apparition du phénomène dans le français parlé en Côte d'Ivoire. La labialisation des voyelles étirées /i ; e ; $\varepsilon ; \tilde{\varepsilon} /$ et la délabialisation des voyelles 
arrondies /y; ə;ø;œ; œ̃/ s'expliquent plutôt par les difficultés articulatoires des éléments de la deuxième série, à savoir les voyelles antérieures arrondies du français.

Le système vocalique du français, avec ses trois voyelles orales internes [ø; ; y], constitue en effet un cas rare (Hagège, $2013: 20$ ). Ces voyelles qui participent de l'originalité de la langue française peuvent être considérées comme difficiles, dans la mesure où « elles réunissent deux mouvements articulatoires rarement associés (articulation antérieure et labialisée) » (Chiss, Filliolet et Maingueneau, 1983 : 94), d'où leur appellation de voyelles orales composées, par opposition aux autres voyelles orales dites simples. On peut dire que la difficulté articulatoire de ces voyelles (rares) combinée à leur absence des systèmes des langues maternelles ivoiriennes fait que les francophones ivoiriens sont comme dans une situation où ils doivent opérer un choix. Ils ont la possibilité de faire l'effort de bien articuler ces voyelles. Dans ce cas, la priorité est à la réalisation de ces voyelles. Ce faisant, il peut y avoir une mise en place anticipée des organes phonatoires en vue de la prononciation desdites voyelles, ou plutôt un retard dans l'abandon de la position des organes phonatoires correspondant à leur prononciation. Cette première possibilité débouche sur des labialisations, et les mécanismes impliqués décrits ici sont ceux à l'origine de l'harmonie vocalique, comme l'expliquent Dubois et al. (1994 : 55). La seconde possibilité semble guidée par la loi du moindre effort. Plutôt que de fournir assez d'énergie pour réaliser ces voyelles difficiles, les locuteurs procèderaient plutôt à une substitution de ces sons par leurs correspondants attestés dans leurs langues maternelles et en français. Dans ce cas, c'est la délabialisation des antérieures arrondies du français. Cela peut expliquer pourquoi en cas de labialisation ou de délabialisation, le choix de la voyelle n'est pas aléatoire : celle qui remplace et celle qui est remplacée se distinguent uniquement par le trait de labialité. Autrement dit, une antérieure étirée est remplacée par l'antérieure arrondie qui lui correspond (ou qui lui est le plus proche), et vice versa. Il semble cependant que les monosyllabiques obéissent beaucoup plus à la loi du moindre effort. Dans « tu »/ty/ et « cœur » $/ \mathrm{k} /$ qui sont souvent réalisés respectivement [ti] et [kع], par exemple, aucune voyelle a priori ne justifie la délabialisation de [y] et [œ]. Dans le cas de «tu », parfois le contexte justifie la délabialisation ou la labialisation. Ainsi, dans «ti es rassasié » [ticrazje], le fait que «tu »/ty/ soit suivi de «es »/E/ dans la forme de base «tu es rassasié»/tyerasazje/, rend prévisible l'assimilation par voisinage (le / $/ \varepsilon$ étiré délabialise /y/, une voyelle arrondie). Dans « ti sais pas pouquoi» [tisepapukwa], l'assimilation se fait par projection de noyau. Cela signifie que la voyelle noyau d'une syllabe influence celle d'une autre syllabe qui lui est plus ou moins proche. Ainsi, le / $/$ / de « sais »/se/ influence le /y/ de /ty/ et le délabialise en lui communiquant son trait de labialité : ici l'assimilation est régressive. C'est la même assimilation par projection de noyau (on parle en réalité de gouvernement par projection nucléaire) qui se passe dans le cas de «meilleur »/mejœ:R/ (ou /mejœ:R/) qui est prononcé [mehe:] en cas de délabialisation de la voyelle antérieure arrondie /œ/ de la seconde syllabe du mot, ou [møhœ:] quand il y a labialisation de la voyelle étirée / $/ \varepsilon /$ (ou /e/), noyau de la première syllabe. Tous ces cas d'assimilation (par voisinage et par projection de noyau) peuvent bien justifier une harmonie vocalique. Il s'agirait ici d'une harmonie d'arrondissement.

L'opposition [o] / [0] montre un autre type d'harmonie vocalique. Le fait que les voyelles des syllabes successives d'un même mot soient identiques, et que l'on n'observe pas d'alternance [o] / [0] au sein d'une même unité lexicale dans le cas de certains mots empruntés aux langues ivoiriennes amène à privilégier la piste de l'harmonie ATR. En effet, dans des mots comme "gbofloto » [gbofloto] ou " gbôlô » [gbolo], le [o] +ATR n'apparait pas dans une syllabe adjacente à une autre ayant pour noyau le [o] -ATR. Mais, étant donné que ces mots sont des emprunts (sans doute aux langues maternelles ivoiriennes), on peut dire qu'ils ont conservé une des propriétés des langues d'origine.

Les nasalisations et les amuïssements constituent des cas marginaux. Ils concernent seulement quelques mots à l'heure actuelle. Les nasalisations sont un autre type d'assimilation 
qui concerne les mots « maman »/mamã/, « jamais »/zame/, « même »/mem/, « moi »/mwa/ et «mais»/mع/. La nasalisation des voyelles orales présentes dans ces mots tient de toute évidence à une influence des consonnes nasales qui les précèdent. La voyelle copie le trait de nasalité de la consonne précédente. La nasalisation est due à une assimilation progressive, c'està-dire qu'il y a un retard dans l'abandon de la position des organes phonatoires correspondant à la prononciation de la nasale (ici [m]). Quant aux amuïssements de « i » dans l'articulation de " président » [prezdã], et de «e » dans la réalisation de "professeur » [profsœ], ils relèvent sans doute d'une négligence dans l'articulation du premier mot, et d'une hypercorrection dans le cas du second. Suivant le schéma de l'hypercorrection, certains Ivoiriens remplacent la voyelle étirée [e] par le schwa [ə] dans « bon appétit » et prononcent ainsi [bõnapti] au lieu de /bõnapeti/.

\section{Conclusion}

Cette étude de la prononciation vise à montrer comment les particularités phonétiques du français parlé en Côte d'Ivoire éclairent la dynamique de la langue française dans ce pays. Nous avons fait un inventaire des sons, et procédé à une analyse de leur fonctionnement.

L'inventaire fait ressortir le rôle des langues maternelles ivoiriennes dans la dynamique du français en Côte d'Ivoire. Les emprunts lexicaux à ces langues peuvent en effet justifier l'extension des systèmes consonantique et vocalique. L'absence de certaines voyelles du français peut s'expliquer par leur absence des bases articulatoires des langues maternelles des participants. Mais il semble que le rôle des langues autochtones se limite principalement à la structure des systèmes consonantique et vocalique. Le fonctionnement des sons révèle, quant à lui, le jeu de lois phonétiques universelles.

Les chutes et les spirantisations observées dans le fonctionnement consonantique mettent en jeu des principe et lois tels que la coarticulation, la loi du moindre effort et la loi des forces consonantiques, qui rendent compte des relâchements, lénitions et chutes de certains sons.

Dans le fonctionnement des voyelles, on observe des délabialisations et labialisations qui sont dues à la combinaison de deux facteurs principaux : les difficultés articulatoires des voyelles antérieures arrondies du français et l'absence de ces voyelles des systèmes des langues maternelles ivoiriennes. Ce faisant, la loi du moindre effort peut expliquer la délabialisation de ces voyelles difficiles. D'un autre côté, l'effort d'une juste prononciation de ces mêmes voyelles aboutit, par assimilation, à la labialisation des voyelles antérieures étirées environnantes. Il en résulte une tendance à l'harmonisation des traits de labialisation de certaines voyelles, ce qui fait penser à une harmonie vocalique. D'autres phénomènes marginaux observables dans le fonctionnement vocalique tels que les amuïssements et les nasalisations relèvent plutôt de la loi du moindre effort, des assimilations et des hypercorrections.

Même s'ils ne sont pas systématiques chez tous les francophones du pays, les faits observés restent assez répandus pour que, selon nous, il soit nécessaire de les prendre en compte dans l'enseignement du français. Cela pourrait aider les enseignants et leurs élèves à savoir (mieux) distinguer les prononciations admises en français standard de référence de celles qui relèvent de pratiques non-standards.

L'étude montre, en fin de compte, que les langues maternelles ivoiriennes ne sont pas entièrement responsables de la dynamique du français en Côte d'Ivoire. Les particularités que nous venons d'analyser se retrouvent chez les locuteurs d'autres variétés du français, aussi bien en France qu'en Amérique du Nord (Québec, Louisiane, etc.). Et, chez les uns comme chez les autres, l'application (sans doute inconsciente) de lois phonétiques universelles est l'une des causes qui sous-tendent les modifications, et par-delà la dynamique du français. 
Abdou Diouf fait observer qu' « il y a un français d'Afrique ; il ne faut pas en avoir honte. Québécois, Belges, Romands font, sans complexes, usage du leur. Il faut cependant lui conserver sa structure et sa solidité » (Organisation internationale de la Francophonie, 2003 : 89). Le français parlé en Côte d'Ivoire est un aspect de ce «français d'Afrique ». Si ses particularités peuvent être expliquées par des lois universelles, on peut alors se demander si cette conception (puriste) d'une langue française monolithique et statique ne devrait pas être dépassée pour voir dans le français parlé en Côte d'Ivoire une variété à part entière qui mérite que l'on s'y intéresse, dans la perspective de son développement.

\section{Bibliographie}

ABOA Abia Alain Laurent, 2008, «La francophonie ivoirienne: enjeux politiques et socioculturels », Documents pour l'histoire du français langue étrangère ou seconde, 40/41, p. 163-178.

ADOPO Assi François, s.d., «Changement tonal et vocalique dans le système verbal de l'akyé », http://www.ltml.ci/files/revue/changement $\% 20 \% 20$ vocalique.pdf, consulté le 10 mai 2019.

AHOUA Firmin et KOUADIO Yao Edouard, 2017, «Caractéristiques acoustiques des consonnes fortis / lenis en potou », Firmin Ahoua et Benjamin Ohi Olugbé, Typologie et documentation des langues en Afrique de l'Ouest. Les actes du $27^{\circ}$ Congrès de la Société de Linguistique de l'Afrique de l'Ouest (SLAO), Paris, L'Harmattan, pp. 107126.

AIMARD Paule, 1996, Les débuts du langage chez l'enfant, Paris, Dunod.

ASSEMOU Assémou Maurice Ludovic, 2017, Les particularités morphosyntaxiques du français de Côte d'Ivoire et la problématique de la norme, Thèse de Doctorat, Abidjan, Université Félix Houphouët-Boigny.

ASSEMOU Assémou Maurice Ludovic, 2018, " Analyse contrastive de la réduplication en français standard et français de Côte d'Ivoire », Studii de gramatica contrastiva/Studies in contrastive grammar, $\mathrm{n}^{\circ} 30$, pp. 7-21.

AZZAM-HANNACHI Radia, 2005, Évolution de l'enseignement des langues vivantes à l'école primaire en France : formation et représentations des enseignants du premier Degré, Thèse de Doctorat, Nancy, Université Nancy 2.

BITTNER Michèle, 1995, Réalisation des constrictives $\int$ et 3 en parler saguenéen, étude acoustique, Mémoire de Maitrise, Université Laval.

BLANCHET Philippe, 2012, La linguistique de terrain. Méthode et théorie. Une approche ethnosociolinguistique de la complexité, Rennes, Presses Universitaires de Rennes.

BOUTIN Akissi Béatrice, 2002, Description de la variation : Étude transformationnelle des phrases du français de Côte d'Ivoire, Thèse de Doctorat, Grenoble, Université de Grenoble.

BOUTIN Akissi Béatrice et TURCSAN Gabor, 2009, «La prononciation du français en Afrique : la Côte d'Ivoire », Jacques Durand, Bertrand Laks, Chantal Lyche, Phonologie, variation et accent du français, Paris, Hermès, pp. 133-156.

CANUT Cécile, 2006, « Lafage Suzanne. Le lexique français de Côte d'Ivoire. Appropriation et créativité », Cahiers d'études africaines, n¹81, pp. 226-229.

CHARRETTE Monik, 1984, "Analyse phonologique des emprunts en dida de Niakassé », Revue québécoise de linguistique, Vol. 14, $\mathrm{n}^{\circ} 1$, pp. 87-111.

CHISS Jean-Louis, FILLIOLET Jacques et MAINGUENAU Dominique, 1983, Linguistique française, Paris, Hachette. 
DODO Jean Claude, s.d., "Quelques faits prosodiques du français standard ivoirien», www.ltml.ci/files/article13/JEAN CLAUDE DODO.pdf, consulté le 02 avril 2019.

DODO Jean Claude, 2014, «La prononciation du français ivoirien : un cas de dynamique linguistique », ANADISS, n¹7, pp. 97-106.

DUBOIS Jean et al., 1994, Dictionnaire de linguistique et des sciences du langage, Paris, Larousse.

DUMONT Pierre, 1990, Le français langue africaine, Paris, L'Harmattan.

EXARE Christelle, 2017, Les aspirations intrusives dans l'anglais des apprenants francophones, Thèse de Doctorat, Paris, Université Sorbonne Nouvelle - Paris 3

HAGEGE Claude, 2013, La structure des langues, $7^{\mathrm{e}}$ édition, Paris, PUF.

HATTIGER Jean-Louis, 1983, Le français populaire d'Abidjan : un cas de pidginisation, Abidjan, ILA.

KABORE Raphaël et TCHAGBALE Zakari, 1998, «ATR, ouverture et arrondissement vocaliques dans quelques systèmes africains », Faits de langues, n¹1-12, pp. 467-490.

KOUADIO N'Guessan Jérémie, 2008, «Le français en Côte d'Ivoire : de l'imposition à l'appropriation décomplexée d'une langue exogène », Documents pour l'histoire $d u$ français langue étrangère ou seconde, 40/41, p. 179-197.

KOUADIO N'Guessan Jérémie, 2006, « Le nouchi et les rapports dioula-français », Le français en Afrique, $\mathrm{n}^{\circ} 21$, p. 177-191.

KOUADIO N'Guessan Jérémie et KOUAME Kouakou, 2004, Parlons baoulé, langue et culture de la Côte d'Ivoire, Paris, L'Harmattan.

KOSSMAN Maarten, 1995, "La spirantisation dans les parlers zénètes : aperçu historique », Petra Bos (ed.), Langues du Maroc : aspects linguistiques dans un contexte minoritaire, Tilburg, Tilburg University Press, p. 11-19.

LAFAGE Suzanne, 1998, "Le français des rues", une variété avancée du français abidjanais », Faits de langues, n॰11-12, p. 135-144.

LAFAGE Suzanne, 2002, « Le lexique français de Côte d'Ivoire. Appropriation et créativité », Le français en Afrique, $\mathrm{n}^{\circ} 16-17$.

LAFKIOUI Mena, 2006, « La spirantisation de la vélaire occlusive simple /k/ dans les variétés berbères du Rif », Studi Maghrébini, p. 219-228.

LÉON Monique et LÉON Pierre, 1997, La prononciation du français, Paris, Nathan.

LYCHE Chantal, 2000, "Affaiblissement consonantique en cadien », Linx, n42, p. 29-43.

MALECOT André, 1977, Contribution à l'étude de la force d'articulation en français, La Haye, Mouton.

MANESSY Gabriel, 1993, «Normes endogènes et français de référence », Daniel Latin, Ambroise Queffelec et Jean-Tabi Manga, Inventaire des usages de la francophonie. Nomenclatures et méthodologies, Montrouge, John-Libbey Eurotext, p. 15-23

MARTINET André, 2008 [1960], Eléments de linguistique générale, $5^{\mathrm{e}}$ édition, Paris, Armand Colin.

MEL Gnamba Bertin et KOUADIO N'Guessan Jérémie, 1990, «Variétés lexicales du français de Côte d'Ivoire », Clas André et Ouoba Benoit, Visages du français, variétés lexicales de l'espace francophone, Montrouge, John Libbey Eurotext, p. 51-58.

MOURIGH Khalid, 2017, « La spirantisation en berbère ghamari », in Vicente Angeles, Caubet Dominique, Amina Naciri-Azzouz (éds.), La région du Nord-Ouest marocain : parlers et pratiques sociales et culturelles, Prensas de la Universidad de Zaragoza, p. 251-263

ORGANISATION INTERNATIONALE DE LA FRANCOPHONIE, 2003, Les États généraux de l'enseignement du français en Afrique subsaharienne. Rapport général, consulté le 23 mai 2016. 
PLOOG Katja, 2001, « Le non-standard entre norme endogène et fantasme d'unicité. L'épopée abidjanaise et sa polémique intrinsèque », Cahiers d'études africaines, n¹63-164, p. 423-442.

RICAUD-DROISY Hélène, OUBRAYRIE-ROUSSEL Nathalie, SAFONT-MOTTAY Claire, 2009, Psychologie du développement, enfance et adolescence, Paris, Dunod.

SIMARD Yves, 1998, " Français de Côte d'Ivoire : principes d'organisation de l'énoncé », Le français en Afrique, ${ }^{\circ} 12$, pp. 295-310.

SIMARD Yves, 1994, "Les français de Côte d'Ivoire ", Langue française, n¹04, p. 20-36.

VOGLER Pierre, "Contribution à une classification des langues kru (Niger-Congo)", http://hal.archives-ouvertes.fr/hal-01574391/document, consulté le 10 mai 2019.

ZOGBO Gnoléba Raymond, 2005, Dictionnaire Bété-Français, Abidjan, Les Editions du CERAP.

\title{
Sources du corpus (Clips vidéo, disponibles sur YouTube)
}

\author{
« Discours mémorable et historique de Gbagbo » \\ « Jeudi, c'est Koulibaly! Le parrainage des candidats à l'élection présidentielle » \\ « Soro Guillaume à ses parents de Ferké : Je ne suis pas un homme qui cède au chantage » \\ «Gnamien Konan : Pourquoi j’ai quitté le RHDP?» \\ « Discours du Président Abdallah Toikeusse MABRI lors de l'Assemblée Générale Constitutive du RHDP » \\ « Sangare : extrait du discours de madame Simone Ehivet Gbagbo - hommage du FPI » \\ « Discours de Koné Mariétou à sa sortie d'audience avec la patronne de l'Onuci (audio)» \\ « Discours de Mabri Toikeusse, président de l'UDPCI » \\ «Ci-présidentielle 2020- Boga Sako sans pitié pour Soro Guillaume » \\ «Bonjour 2016 prestation de Joel et Agalawal». \\ «Bonjour 2017 prestation de magnifique » \\ « Garba 50 - C'est L'Argent qui fait tout» \\ « Garba 50 - Survivant ». \\ «Garba 50- Sans soi (Wollosso)» \\ «Gbazé Thérèse et sa rivale Ange font la paix et appellent les Ivoiriens à la réconciliation » \\ «Gbazé Thérèse et Ange font palabre» \\ « Allons à Wassakara - Billy Billy », \\ « Billy Billy Ma lettre au président »
}




\section{GLOTTOPOL}

Revue de sociolinguistique en ligne

Comité de rédaction : Michaël Abecassis, Salih Akin, Sophie Babault, Claude Caitucoli, Véronique Castellotti, Régine Delamotte, Robert Fournier, Stéphanie Galligani, Emmanuelle Huver, Normand Labrie, Foued Laroussi, Benoit Leblanc, Fabienne Leconte, Gudrun Ledegen, Danièle Moore, Clara Mortamet, Alioune Ndao, Isabelle Pierozak, Gisèle Prignitz.

Rédactrice en chef : Clara Mortamet.

Comité scientifique : Claudine Bavoux, Michel Beniamino, Jacqueline Billiez, Philippe Blanchet, Pierre Bouchard, Ahmed Boukous, Pierre Dumont, Jean-Michel Eloy, Françoise Gadet, Monica Heller, Caroline Juilliard, Jean-Marie Klinkenberg, Jean Le Du, Marinette Matthey, Jacques Maurais, Marie-Louise Moreau, Robert Nicolaï, Didier de Robillard, Paul Siblot, Claude Truchot, Daniel Véronique.

\section{Comité de lecture pour ce numéro :}

Mickael Abecassis, Michelle Auzanneau, Salih Akin, Céline Amourette, Gabriel Bergounioux, Philippe Boula de Mareuil, Catherine Brissaud, Aude Bretegnier, Maria Candea, Véronique Castellotti, Régine Delamotte, Jean-François De Pietro, Catherine Delarue-Breton, Anne Dister, Alexandre Duchêne, Valentin Feussi, Françoise Gadet, Stéphanie Galligani, François Gaudin, Monica Heller, Emmanuelle Huver, Caroline Juilliard, Abou Bakri Kebe, Jean-Marie Klinkenberg, Christian Lagarde, Foued Laroussi, Fabienne Leconte, Evelyne Lloze, Nolwenn Lorenzi, Gudrun Ledegen, Nadja Maillard, Marinette Matthey, Bruno Maurer, Véronique Miguel Addisu, Saskia Mugnier, Claire Oger, Marielle Rispail, Richard Sabria, Véronique Traverso, Christel Troncy, Cécile Van den Avenne. 